\title{
Spatial distribution of the persistent organic pollutants across the Tibetan Plateau and its linkage with the climate systems: a 5-year air monitoring study
}

Xiaoping Wang et al.

Correspondence to: Xiaoping Wang (wangxp@itpcas.ac.cn)

The copyright of individual parts of the supplement might differ from the CC-BY 3.0 licence. 
Summary of the number of pages, figures, and tables

\begin{tabular}{|c|c|c|}
\hline Text S1 & Sampling program of the active air sampling & Page S3 \\
\hline Text S2 & $\begin{array}{c}\text { Chemical extraction, cleanup and details on the gas } \\
\text { chromatographic temperature }\end{array}$ & Page S4 \\
\hline Table S1 & $\begin{array}{l}\text { Data of field blank concentrations and method } \\
\text { detection limits (MDL, ng/sample) for each sampling } \\
\text { year }\end{array}$ & Page S5 \\
\hline Table S2 & $\begin{array}{l}\text { Concentration presented as amounts of POPs } \\
\text { sequestered over the sampling period (ng/sampler) }\end{array}$ & Page S6 \\
\hline Table S3 & $\begin{array}{c}\text { The concentrations of duplicates (ng/sample) and } \\
\text { average RSD for each compound }\end{array}$ & Page S10 \\
\hline Table S4 & $\begin{array}{l}\text { Estimated Volumetric Air Concentrations }\left(\mathrm{pg} \mathrm{m}^{-3}\right) \\
\text { from the Tibetan Plateau Sites }\end{array}$ & Page S12 \\
\hline Text S3 & The comparison between AAS and PAS & Page S16 \\
\hline Table S5 & $\begin{array}{l}\text { Average air concentrations (ng/sample) for each } \\
\text { sampling site during the } 5 \text { sampling years }\end{array}$ & Page S17 \\
\hline Table S6 & $\begin{array}{l}\text { Average volumetric concentrations }\left(\mathrm{pg} / \mathrm{m}^{3}\right) \text { for each } \\
\text { sampling site during the } 5 \text { sampling years }\end{array}$ & Page S18 \\
\hline
\end{tabular}




\begin{tabular}{|c|c|c|}
\hline Text S4 & $\begin{array}{l}\text { Comparison between concentrations of the current } \\
\text { study and GAPS study }\end{array}$ & Page S19 \\
\hline Table S7 & $\begin{array}{l}\text { Randomized block ANOVA test for the concentrations } \\
\text { from different sampling sites }\end{array}$ & Page S20 \\
\hline Text S5 & $\begin{array}{l}\text { Calculation equations for fugacity, fugacity ratio and } \\
\text { fugacity fluxes }\end{array}$ & Page S23 \\
\hline Table S8 & $\begin{array}{l}\text { The soil-air equilibrium status }(f f) \text { of Tibetan Plateau } \\
\text { sites. }\end{array}$ & Page S24 \\
\hline Table S9 & $\begin{array}{l}\text { The soil-air net volatilization fluxes }\left(\mathrm{ng} / \mathrm{m}^{2} / \mathrm{h}\right) \text { of PCB- } \\
\text { 28, PCB-52, HCB, } \alpha-\mathrm{HCH} \text { and } \gamma-\mathrm{HCH} \text { at the Tibetan } \\
\text { Plateau sites. }\end{array}$ & Page S26 \\
\hline Table S10 & Relative composition data (\%) used for cluster analysis & Page S28 \\
\hline Table S11 & Results of the grouping & Page S30 \\
\hline Table S12 & The representative sites in each group & Page S31 \\
\hline Figure S1 & $\begin{array}{l}\text { Result of cluster analysis after including the duplicate } \\
\text { samples }\end{array}$ & Page S32 \\
\hline Figure S2 & Inter-annual concentration variation from 2007 to 2012 & Page S34 \\
\hline Table S13 & $\begin{array}{l}\text { Results of randomized block ANOVA test for the } \\
\text { concentrations of DDTs in the monsoon region, westerly } \\
\text { region and transition region from } 2007 \text { to } 2012 \text {. (p < } \\
0.05 \text { indicates that the levels of DDTs significantly differ } \\
\text { between two years.) }\end{array}$ & Page S35 \\
\hline References & & Page S36 \\
\hline
\end{tabular}




\section{Text S1 Sampling program of the active air sampling}

23 The sampling period was from November 2008 to September 2011, and the samples were collected every 2 weeks. Polyurethane foam (PUF, 7.5

$24 \mathrm{~cm} \times 6 \mathrm{~cm}$ diameter) was used to collect the gas-phase OCPs and PCBs. The particulates were collected on glass fiber filters. A pair of PUF

25 plugs $(7.5 \mathrm{~cm} \times 3 \mathrm{~cm})$ was installed in series inside the sampling cylinder to monitor potential breakthrough. Typically, $500-700 \mathrm{~m}^{3}$ of air was

26 collected over a two-week period. Before sampling, all of the PUF plugs were pre-cleaned by a Soxhlet extraction using dichloromethane

27 (DCM) for 24 hours and the filters were baked at $450{ }^{\circ} \mathrm{C}$ for 5 hours. After sampling, the harvested PUF plugs and filters were stored in sealed,

28 solvent-rinsed, glass jars and stored at $-20{ }^{\circ} \mathrm{C}$ until extraction. 


\section{Text S2 Chemical extraction, cleanup and details on the gas chromatographic temperature}

The retrieved XAD sample was transferred into the Soxhlet body and spiked with the recovery standards of 2,4,5,6-tetrachloro-m-xylene (TCmX), and decachlorobiphenyl (PCB-209). Samples were Soxhlet-extracted using DCM for 24 hours. The extracts were first concentrated by a rotary evaporator and solvent exchanged to hexane. Then, the samples were loaded on the top of a chromatography column (from the top to bottom: $1 \mathrm{~g}$ of anhydrous sodium sulfate, $2 \mathrm{~g}$ of $3 \%$ deactivated alumina, and $3 \mathrm{~g}$ of $6 \%$ deactivated silica gel). The column was eluted with $30 \mathrm{~mL}$ of a mixture of DCM and hexane (1:1). The elute was further cleaned using gel-permeation chromatography (GPC, containing $6 \mathrm{~g}$ of Biobeads SX3), and the samples were finally solvent exchanged and concentrated in $20 \mu \mathrm{L}$ of dodecane containing a known quantity of pentachloronitrobenzene (PCNB) and PCB 54 as the internal standards. Details on the gas chromatographic temperature are given in text S2 (Supporting Information).

The samples were analyzed on a Finnigan-TRACE GC-MS system with a CP-Sil 8CB capillary column (50 m, $0.25 \mathrm{~mm}, 0.25 \mu \mathrm{m}$ ), operating under single-ion

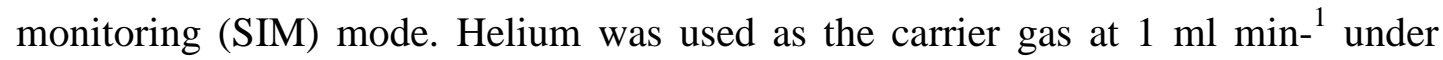
constant-flow mode. The oven temperature began at $100{ }^{\circ} \mathrm{C}$ for 2 min and was increased at a rate of $20^{\circ} \mathrm{C} \min ^{-1}$ to $140{ }^{\circ} \mathrm{C}$, then increased at a rate of $4{ }^{\circ} \mathrm{C} \mathrm{min}-1$ to $200{ }^{\circ} \mathrm{C}$, held for $10 \mathrm{~min}$, and finally increased at a rate of $4{ }^{\circ} \mathrm{C} \min ^{-1}$ to $300{ }^{\circ} \mathrm{C}$ and held for $17 \mathrm{~min}$. 
Table S1. Data of field blank concentrations and method detection limits (MDL,

ng/sample) for each sampling year

\begin{tabular}{|c|c|c|c|c|c|c|c|c|}
\hline $\begin{array}{c}\text { Sample } \\
\text { name }\end{array}$ & $\alpha-\mathrm{HCH}$ & $\gamma-\mathbf{H C H}$ & HCB & $\begin{array}{l}\text { o,p'- } \\
\text { DDE }\end{array}$ & $\begin{array}{l}\text { p,p'- } \\
\text { DDE }\end{array}$ & $\begin{array}{l}\text { o,p'- } \\
\text { DDT }\end{array}$ & $\begin{array}{l}\text { p,p'- } \\
\text { DDT }\end{array}$ & $\sum$ PCBs \\
\hline 2007-08 blank ${ }^{\mathrm{a}}$ & 0.27 & 0.68 & ND & 0.44 & 0.34 & ND & ND & 0.19 \\
\hline $2007-08 \mathrm{MDL}^{\mathrm{a}}$ & 0.28 & 1.2 & 0.03 & 0.47 & 0.36 & 0.03 & 0.03 & 0.18 \\
\hline 2008-09 blank1 & 0.01 & 0.01 & 0.78 & ND & 0.21 & ND & ND & 0.16 \\
\hline 2008-09 blank2 & ND & 0.02 & 0.39 & 0.12 & 0.23 & ND & ND & 0.12 \\
\hline 2008-09 blank3 & ND & ND & 0.16 & ND & 0.14 & 0.28 & ND & 0.08 \\
\hline 2008-09 blank4 & ND & ND & 0.35 & 0.02 & 0.25 & ND & ND & 0.14 \\
\hline 2008-09 blank5 & ND & 0.10 & 0.33 & 0.14 & ND & ND & ND & 0.13 \\
\hline Blank average & 0.01 & 0.04 & 0.40 & 0.09 & 0.21 & 0.28 & ND & 0.13 \\
\hline 2008-09 MDL & 0.20 & 0.19 & 1.09 & 0.29 & 0.35 & 0.20 & $0.20^{\mathrm{b}}$ & 0.21 \\
\hline 1/2 MDL & 0.1 & 0.1 & 1.55 & 0.15 & 0.17 & 0.1 & 0.1 & 0.1 \\
\hline 2009-10 blank 1 & ND & ND & 0.42 & ND & 0.40 & ND & 0.41 & 0.05 \\
\hline 2009-10 blank 2 & ND & ND & 0.63 & ND & 0.26 & ND & ND & 0.11 \\
\hline 2009-10 blank 3 & ND & ND & 0.55 & ND & 0.27 & ND & ND & 0.08 \\
\hline 2009-10 blank 4 & 0.42 & & 0.26 & 0.13 & 0.39 & 0.23 & 0.53 & 0.07 \\
\hline 2009-10 blank 5 & 0.05 & 0.04 & 0.51 & ND & 0.29 & ND & 0.25 & 0.09 \\
\hline Blank average & 0.24 & 0.09 & 0.47 & 0.13 & 0.32 & 0.23 & 0.40 & 0.08 \\
\hline 2009-10 MDL & 1.02 & 0.30 & 0.90 & 0.20 & 0.52 & 0.20 & 0.82 & 0.15 \\
\hline 1/2 MDL & 0.5 & 0.15 & 0.45 & 0.1 & 0.26 & 0.1 & 0.41 & 0.08 \\
\hline 2010-11 blank 1 & 0.05 & 0.03 & 0.37 & ND & 0.22 & ND & 0.19 & 0.24 \\
\hline 2010-11 blank 2 & 0.02 & 0.02 & 0.22 & 0.01 & 0.10 & 0.03 & 0.02 & 0.11 \\
\hline 2010-11 blank 3 & 0.08 & & 0.47 & $\mathrm{ND}$ & 0.17 & 0.07 & 0.18 & 0.16 \\
\hline 2010-11 blank 4 & 0.06 & 0.06 & 0.33 & ND & 0.26 & 0.19 & 0.15 & 0.17 \\
\hline 2010-11 blank 5 & 0.05 & 0.07 & 0.59 & ND & 0.24 & ND & 0.18 & 0.15 \\
\hline 2010-11 blank 6 & 0.03 & 0.05 & 0.40 & ND & 0.09 & 0.06 & 0.05 & 0.08 \\
\hline Blank average & 0.05 & 0.05 & 0.40 & 0.01 & 0.18 & 0.09 & 0.13 & 0.15 \\
\hline 2010-11 MDL & 0.12 & 0.10 & 0.77 & 0.20 & 0.40 & 0.30 & 0.35 & 0.32 \\
\hline 1/2 MDL & 0.06 & 0.05 & 0.38 & 0.1 & 0.2 & 0.15 & 0.17 & 0.16 \\
\hline 2011-12 blank 1 & 0.04 & ND & 0.29 & 0.01 & 0.07 & 0.03 & 0.04 & 0.04 \\
\hline 2011-12 blank 2 & 0.04 & ND & 0.27 & ND & 0.08 & 0.02 & 0.04 & 0.06 \\
\hline 2011-12 blank 3 & 0.03 & ND & 0.23 & 0.02 & 0.08 & 0.02 & 0.04 & 0.03 \\
\hline 2011-12 blank 4 & 0.05 & 0.03 & 0.39 & 0.01 & 0.09 & 0.07 & 0.01 & 0.07 \\
\hline 2011-12 blank 5 & 0.07 & 0.04 & 0.61 & 0.01 & 0.05 & 0.17 & 0.19 & 0.05 \\
\hline 2011-12 blank 6 & 0.03 & 0.01 & 0.26 & 0.02 & 0.09 & 0.16 & 0.16 & 0.02 \\
\hline Blank average & 0.04 & 0.03 & 0.34 & 0.01 & 0.08 & 0.08 & 0.08 & 0.05 \\
\hline 2011-12 MDL & 0.09 & 0.07 & 0.76 & 0.04 & 0.12 & 0.29 & 0.30 & 0.10 \\
\hline 1/2 MDL & 0.05 & 0.04 & 0.4 & 0.02 & 0.06 & 0.15 & 0.15 & 0.05 \\
\hline
\end{tabular}

The methods detection limits (MDLs) were derived as the mean field blank

58 concentration plus 3 times of its standard deviation.

a: The data have been reported in Wang et al. 2010. 
Table S2 Concentration presented as amounts of POPs sequestered over the sampling period (ng/sampler)

\begin{tabular}{|c|c|c|c|c|c|c|c|c|c|c|c|c|c|c|}
\hline Sampling site & $\alpha-Н С Н$ & $\gamma$-HCH & НСВ & $\begin{array}{l}\text { o,p'- } \\
\text { DDE }\end{array}$ & $\begin{array}{l}\text { p,p'- } \\
\text { DDE }\end{array}$ & $\begin{array}{l}\text { o,p'- } \\
\text { DDT }\end{array}$ & $\begin{array}{l}\text { p,p'- } \\
\text { DDT }\end{array}$ & $\begin{array}{c}\text { PCB- } \\
28\end{array}$ & $\begin{array}{c}\text { PCB- } \\
52\end{array}$ & $\begin{array}{c}\text { PCB- } \\
101\end{array}$ & $\begin{array}{c}\text { PCB- } \\
153\end{array}$ & $\begin{array}{c}\text { PCB- } \\
138\end{array}$ & $\begin{array}{c}\text { PCB- } \\
180\end{array}$ & $\sum_{6}$ PCBs \\
\hline \multicolumn{15}{|c|}{$2007-2008$} \\
\hline Xigaze & 3.1 & 1.2 & 24.7 & 3.1 & 4.9 & 26 & 10.7 & 0.51 & 0.48 & 0.34 & 0.42 & 1.04 & $N D$ & 2.81 \\
\hline Nam co & 0.1 & 0.6 & 2.5 & 2.3 & 0.18 & 2.9 & 0.5 & 0.1 & 0.06 & 0.02 & 0.07 & 0.18 & $N D$ & 0.45 \\
\hline Lhasa & 4.1 & 1.4 & 56.2 & 2.2 & 5.7 & 22.3 & 6.2 & 0.54 & 0.15 & 0.02 & 0.23 & 0.54 & $N D$ & 1.5 \\
\hline Lhaze & 1.6 & 0.8 & 22 & 1 & 1 & 9.4 & 2.5 & 0.4 & 0.14 & 0.11 & 0.08 & 0.2 & $N D$ & 0.95 \\
\hline Lulang & 1.6 & 0.5 & 16.9 & 2.9 & 3.9 & 28.5 & 6.5 & 0.53 & 0.44 & 0.02 & 0.12 & 0.6 & $N D$ & 1.73 \\
\hline Everest & 4.5 & 5.4 & 22 & 0.1 & 0.3 & 3.1 & 0.5 & 2.1 & 0.1 & 0.02 & 0.02 & 0.02 & $N D$ & 2.28 \\
\hline Naqu & 7.3 & 1.9 & 27.4 & 1.7 & 0.8 & 15.3 & 4.1 & 0.28 & 0.25 & 0.1 & 0.07 & 0.65 & $N D$ & 1.37 \\
\hline Qamdo & 1.2 & 1.1 & 16.1 & 6.7 & 11 & 34.4 & 9.4 & 1.06 & 0.22 & 0.02 & 0.21 & 1.04 & $N D$ & 2.57 \\
\hline Saga & 4.9 & 1.6 & 20.2 & 3.2 & 5.7 & 28.5 & 9 & 0.4 & 0.3 & 0.16 & 0.08 & 0.25 & $N D$ & 1.21 \\
\hline Rawu & 16.4 & 18.7 & 11.8 & 1.1 & 4.1 & 38.9 & 7.2 & 2.38 & 0.17 & 0.02 & 0.02 & 0.02 & $N D$ & 2.63 \\
\hline Bomi & 6.5 & 3.6 & 60.7 & 2 & 4.2 & 11.5 & 3 & 0.89 & 0.48 & 0.02 & 0.02 & 1.38 & $N D$ & 2.81 \\
\hline GBJD & 6.9 & 2.1 & 23.1 & 3.4 & 6.8 & 28.1 & 6.5 & 0.4 & 0.23 & 0.02 & 0.26 & 0.93 & $N D$ & 1.86 \\
\hline Golmud & 2.9 & 1 & 44.2 & 1.5 & 1.2 & 1.4 & 0.5 & 0.63 & 0.27 & 0.02 & 0.02 & 0.02 & $N D$ & 0.98 \\
\hline Gar & 3.7 & 13.0 & 8.4 & 0.9 & 1.9 & 9.3 & 2.1 & 1.25 & 0.11 & 0.02 & 0.02 & 0.02 & $N D$ & 1.44 \\
\hline Chayu & / & / & I & / & / & / & / & / & I & / & I & I & / & I \\
\hline Muztagata & / & / & I & / & / & / & / & l & l & / & / & / & l & / \\
\hline \multicolumn{15}{|c|}{ 2008-2009 } \\
\hline Xigaze & 6.63 & 2.59 & 12.06 & 0.97 & 1.33 & 2.55 & 3.28 & 0.06 & 0.41 & 0.12 & 0.03 & 0.05 & $N D$ & 0.69 \\
\hline Nam co & 3.61 & 1.32 & 26.45 & 0.31 & 0.36 & 1.33 & 0.99 & 0.22 & 0.62 & 0.12 & 0.07 & 0.05 & $N D$ & 1.09 \\
\hline Lhasa & 4.96 & 2.91 & 29.92 & 0.15 & 0.93 & 4.85 & 2.44 & 0.22 & 0.54 & 0.12 & 0.01 & 0.05 & $N D$ & 0.95 \\
\hline Lhaze & 4.35 & 1.07 & 18.66 & 0.15 & 0.51 & 2.26 & 2.50 & 0.22 & 0.70 & 0.12 & 0.04 & 0.11 & $N D$ & 1.22 \\
\hline Lulang & 2.89 & 0.88 & 8.49 & 0.84 & 0.80 & 4.66 & 5.61 & 0.06 & 0.37 & 0.12 & 0.02 & 0.05 & $N D$ & 0.63 \\
\hline
\end{tabular}




\begin{tabular}{|c|c|c|c|c|c|c|c|c|c|c|c|c|c|c|}
\hline Everest & 5.01 & 1.73 & 24.24 & 0.15 & 0.55 & 5.83 & 4.72 & 0.11 & 0.47 & 0.12 & 0.01 & 0.05 & $N D$ & 0.79 \\
\hline Naqu & 11.01 & 2.64 & 12.56 & 0.15 & 0.17 & 1.18 & 2.12 & 0.06 & 0.39 & 0.12 & 0.03 & 0.05 & $N D$ & 0.67 \\
\hline Qamdo & 2.23 & 0.89 & 11.08 & 0.15 & 1.26 & 0.40 & 2.15 & 0.21 & 0.56 & 0.12 & 0.06 & 0.05 & $N D$ & 1.04 \\
\hline Saga & 4.66 & 1.32 & 14.54 & 0.15 & 0.96 & 3.14 & 3.39 & 0.19 & 0.50 & 0.12 & 0.03 & 0.05 & $N D$ & 0.91 \\
\hline Rawu & 1.65 & 0.34 & 6.32 & 0.15 & 0.17 & 0.70 & 1.58 & 0.06 & 0.13 & 0.12 & 0.01 & 0.05 & $N D$ & 0.38 \\
\hline Bomi & 14.00 & 3.91 & 26.95 & 0.60 & 4.14 & 9.65 & 21.91 & 0.15 & 0.49 & 0.12 & 0.03 & 0.05 & $N D$ & 0.87 \\
\hline GBJD & I & / & I & I & I & I & I & I & I & / & I & I & & / \\
\hline Golmud & 4.84 & 2.30 & 33.85 & 0.15 & 0.17 & 0.10 & 0.10 & 0.25 & 0.70 & 0.12 & 0.01 & 0.05 & $N D$ & 1.14 \\
\hline Gar & 6.55 & 1.98 & 30.14 & 0.65 & 0.97 & 0.10 & 1.07 & 0.27 & 0.74 & 0.12 & 0.03 & 0.05 & $N D$ & 1.22 \\
\hline Chayu & 3.34 & 0.84 & 16.10 & 0.15 & 1.09 & 5.97 & 15.54 & 0.06 & 0.30 & 0.12 & 0.01 & 0.05 & $N D$ & 0.55 \\
\hline Muatagata & I & / & I & l & I & I & I & I & I & 1 & I & I & / & l \\
\hline \multicolumn{15}{|c|}{$2009-2010$} \\
\hline Xigaze & 2.03 & 0.63 & 9.83 & 3.16 & 4.70 & 8.97 & 3.85 & 0.07 & 0.02 & 0.14 & 0.02 & 0.05 & $N D$ & 0.31 \\
\hline Nam co & 3.14 & 0.84 & 15.98 & 0.66 & 1.46 & 11.46 & 6.37 & 0.07 & 0.12 & 0.70 & 0.16 & 0.22 & $N D$ & 1.36 \\
\hline Lhasa & 1.73 & 0.53 & 9.99 & 0.42 & 2.64 & 7.74 & 3.46 & 0.07 & 0.05 & 0.28 & 0.02 & 0.09 & $N D$ & 0.52 \\
\hline Lhaze & 3.88 & 1.33 & 11.52 & 0.10 & 1.56 & 4.53 & 3.20 & 0.07 & 0.05 & 0.20 & 0.02 & 0.05 & $N D$ & 0.40 \\
\hline Lulang & 3.10 & 0.75 & 8.50 & 0.35 & 2.07 & 11.73 & 5.81 & 0.07 & 0.06 & 0.17 & 0.02 & 0.07 & $N D$ & 0.41 \\
\hline Everest & 4.15 & 1.24 & 14.37 & 0.59 & 1.98 & 11.21 & 4.88 & 0.07 & 0.09 & 0.24 & 0.02 & 0.04 & $N D$ & 0.47 \\
\hline Naqu & 10.78 & 2.44 & 15.33 & 0.10 & 1.07 & 4.33 & 1.95 & 0.07 & 0.06 & 0.14 & 0.02 & 0.03 & $N D$ & 0.33 \\
\hline Qamdo & 2.99 & 1.18 & 11.58 & 0.23 & 1.86 & 3.24 & 2.67 & 0.16 & 0.16 & 0.21 & 0.02 & 0.02 & $N D$ & 0.58 \\
\hline Saga & 2.52 & 0.65 & 9.58 & 0.10 & 1.89 & 4.71 & 4.73 & 0.07 & 0.05 & 0.15 & 0.02 & 0.02 & $N D$ & 0.32 \\
\hline Rawu & 3.89 & 0.97 & 15.70 & 0.22 & 1.34 & 7.19 & 3.67 & 0.07 & 0.06 & 0.11 & 0.02 & 0.02 & $N D$ & 0.29 \\
\hline Bomi & 6.92 & 2.16 & 7.31 & 1.23 & 15.05 & 26.49 & 21.70 & 0.25 & 0.02 & 0.32 & 0.02 & 0.06 & $N D$ & 0.68 \\
\hline GBJD & l & / & l & l & I & / & / & / & / & I & 1 & / & I & / \\
\hline Golmud & 6.03 & 1.59 & 33.31 & 0.20 & 1.22 & 5.15 & 1.74 & 0.24 & 0.28 & 0.32 & 0.02 & 0.08 & $N D$ & 0.95 \\
\hline Gar & 1.27 & 0.86 & 13.20 & 0.10 & 1.01 & 1.49 & 1.96 & 0.07 & 0.08 & 0.27 & 0.02 & 0.12 & $N D$ & 0.57 \\
\hline Chayu & 6.70 & 1.83 & 26.73 & 1.43 & 7.45 & 31.77 & 17.19 & 0.18 & 0.14 & 0.31 & 0.02 & 0.05 & $N D$ & 0.71 \\
\hline
\end{tabular}




\begin{tabular}{|c|c|c|c|c|c|c|c|c|c|c|c|c|c|c|}
\hline Muztaga & 1.83 & 0.37 & 16.28 & 0.10 & 1.00 & 1.01 & 1.30 & 0.07 & 0.02 & 0.17 & 0.02 & 0.02 & $N D$ & 0.31 \\
\hline \multicolumn{15}{|c|}{$2010-2011$} \\
\hline Xigaze & 1.62 & 0.24 & 11.64 & 0.54 & 1.41 & 0.92 & 0.18 & 0.12 & 0.04 & 0.07 & 0.02 & 0.05 & $N D$ & 0.31 \\
\hline Nam co & 1.13 & 0.17 & 8.54 & 0.10 & 0.20 & 0.83 & 0.63 & 0.35 & 0.04 & 0.07 & 0.02 & 0.05 & $N D$ & 0.54 \\
\hline Lhasa & 0.73 & 0.11 & 5.27 & 0.24 & 0.99 & 1.16 & 0.59 & 0.12 & 0.04 & 0.07 & 0.02 & 0.05 & $N D$ & 0.31 \\
\hline Lhaze & 1.23 & 0.17 & 9.90 & 0.10 & 0.20 & 0.36 & 0.18 & 0.12 & 0.04 & 0.07 & 0.02 & 0.05 & $N D$ & 0.31 \\
\hline Lulang & 1.41 & 0.16 & 7.39 & 0.24 & 0.71 & 2.06 & 0.93 & 0.12 & 0.04 & 0.07 & 0.02 & 0.05 & $N D$ & 0.31 \\
\hline Everest & 2.30 & 0.55 & 22.23 & 0.28 & 0.73 & 2.39 & 0.87 & 0.12 & 0.08 & 0.07 & 0.02 & 0.05 & $N D$ & 0.35 \\
\hline Naqu & 6.04 & 1.07 & 12.83 & 0.10 & 0.20 & 0.71 & 0.18 & 0.12 & 0.04 & 0.07 & 0.02 & 0.05 & $N D$ & 0.31 \\
\hline Qamdo & 1.36 & 0.22 & 9.03 & 0.10 & 0.41 & 0.44 & 0.18 & 0.12 & 0.04 & 0.07 & 0.02 & 0.05 & $N D$ & 0.31 \\
\hline Saga & 3.42 & 0.54 & 16.28 & 0.10 & 0.67 & 0.95 & 1.06 & 0.12 & 0.04 & 0.07 & 0.02 & 0.05 & $N D$ & 0.31 \\
\hline Rawu & 1.53 & 0.33 & 7.11 & 0.21 & 0.55 & 1.26 & 0.41 & 0.38 & 0.04 & 0.07 & 0.02 & 0.05 & $N D$ & 0.57 \\
\hline Bomi & 3.83 & 0.58 & 5.05 & 0.26 & 4.13 & 1.96 & 1.12 & 0.12 & 0.04 & 0.07 & 0.02 & 0.05 & $N D$ & 0.31 \\
\hline GBJD & 3.59 & 0.84 & 9.04 & 0.25 & 1.08 & 3.28 & 1.50 & 0.12 & 0.04 & 0.07 & 0.02 & 0.05 & $N D$ & 0.31 \\
\hline Golmud & 1.28 & 0.17 & 19.88 & 0.10 & 0.20 & 0.33 & 0.18 & 0.12 & 0.07 & 0.07 & 0.02 & 0.05 & $N D$ & 0.34 \\
\hline Gar & 0.67 & 0.11 & 8.97 & 0.10 & 0.20 & 0.15 & 0.18 & 0.12 & 0.04 & 0.07 & 0.02 & 0.05 & $N D$ & 0.31 \\
\hline Chayu & 1.31 & 0.16 & 9.02 & 0.66 & 4.94 & 8.06 & 5.36 & 0.12 & 0.04 & 0.07 & 0.02 & 0.05 & $N D$ & 0.31 \\
\hline Muztaga & 2.14 & 0.22 & 25.31 & 0.10 & 0.90 & 0.15 & 0.18 & 0.12 & 0.09 & 0.07 & 0.02 & 0.05 & $N D$ & 0.36 \\
\hline \multicolumn{15}{|c|}{$2011-2012$} \\
\hline Xigaze & 1.10 & 0.27 & 6.71 & 0.31 & 0.78 & 1.56 & 0.77 & 0.03 & 0.03 & 0.05 & 0.01 & 0.01 & $N D$ & 0.14 \\
\hline Nam co & 1.31 & 0.34 & 10.07 & 0.09 & 0.22 & 1.98 & 0.75 & 0.03 & 0.03 & 0.05 & 0.01 & 0.02 & $N D$ & 0.15 \\
\hline Lhasa & 0.60 & 0.14 & 3.65 & 0.31 & 1.39 & 2.60 & 1.65 & 0.03 & 0.02 & 0.12 & 0.02 & 0.03 & $N D$ & 0.23 \\
\hline Lhaze & 1.15 & 0.21 & 5.77 & 0.10 & 0.37 & 1.51 & 1.03 & 0.03 & 0.02 & 0.05 & 0.02 & 0.02 & $N D$ & 0.15 \\
\hline Lulang & 1.75 & 0.42 & 5.22 & 0.29 & 0.89 & 5.33 & 3.15 & 0.03 & 0.04 & 0.05 & 0.01 & 0.02 & $N D$ & 0.16 \\
\hline Everest & 1.80 & 0.56 & 11.97 & 0.22 & 0.75 & 4.11 & 2.29 & 0.03 & 0.05 & 0.12 & 0.02 & 0.03 & $N D$ & 0.26 \\
\hline Naqu & 6.48 & 1.45 & 14.72 & 0.13 & 0.39 & 1.73 & 1.58 & 0.06 & 0.06 & 0.05 & 0.02 & 0.02 & $N D$ & 0.22 \\
\hline Qamdo & 0.83 & 0.25 & 4.08 & 0.12 & 0.64 & 0.96 & 0.79 & 0.03 & 0.03 & 0.11 & 0.02 & 0.03 & $N D$ & 0.23 \\
\hline
\end{tabular}




\begin{tabular}{|c|c|c|c|c|c|c|c|c|c|c|c|c|c|c|}
\hline Saga & 1.23 & 0.37 & 6.45 & 0.09 & 0.29 & 0.97 & 0.53 & 0.03 & 0.03 & 0.05 & 0.01 & 0.01 & $N D$ & 0.14 \\
\hline Rawu & 1.09 & 0.29 & 4.46 & 0.19 & 0.52 & 2.81 & 1.60 & 0.03 & 0.03 & 0.05 & 0.01 & 0.02 & $N D$ & 0.15 \\
\hline Bomi & 1.13 & 0.28 & 2.76 & 0.40 & 2.95 & 5.47 & 3.79 & 0.03 & 0.01 & 0.15 & 0.08 & 0.11 & $N D$ & 0.39 \\
\hline GBJD & 2.19 & 0.48 & 7.36 & 0.27 & 1.14 & 5.11 & 22.68 & 0.06 & 0.04 & 0.13 & 0.01 & 0.02 & $N D$ & 0.27 \\
\hline Golmud & 1.97 & 0.73 & 18.98 & 0.05 & 0.49 & 0.41 & 0.56 & 0.14 & 0.08 & 0.20 & 0.02 & 0.05 & $N D$ & 0.50 \\
\hline Gar & 0.82 & 0.21 & 6.82 & 0.04 & 0.19 & 0.44 & 0.35 & 0.03 & 0.03 & 0.05 & 0.01 & 0.01 & $N D$ & 0.14 \\
\hline Chayu & 1.56 & 0.31 & 6.12 & 0.41 & 2.34 & 7.82 & 6.37 & 0.03 & 0.03 & 0.11 & 0.02 & 0.02 & $N D$ & 0.22 \\
\hline Muztagata & 2.25 & 0.33 & 22.00 & 0.02 & 1.20 & 0.80 & 1.30 & 0.03 & 0.02 & 0.15 & 0.02 & 0.01 & $N D$ & 0.24 \\
\hline
\end{tabular}

\section{Entries in italics are substituted values at 1/2 MDL.}

If the concentration of a compound after blank correction was below the MDL, the concentration was substituted with 1/2 MDL.

PCB 180 was never detected in samples 
Table S3. The concentrations of duplicates (ng/sample) and average RSD for each compound

\begin{tabular}{|c|c|c|c|c|c|c|c|c|c|}
\hline $\begin{array}{c}\text { Sampling } \\
\text { year }\end{array}$ & $\begin{array}{c}\text { Sample } \\
\text { name }\end{array}$ & $\alpha-\mathrm{HCH}$ & $\gamma-\mathbf{H C H}$ & HCB & $\begin{array}{l}\text { o,p'- } \\
\text { DDE }\end{array}$ & $\begin{array}{l}\text { p,p'- } \\
\text { DDE }\end{array}$ & $\begin{array}{l}\text { o,p'- } \\
\text { DDT }\end{array}$ & $\begin{array}{l}\text { p,p'- } \\
\text { DDT }\end{array}$ & $\sum$ PCBs \\
\hline \multirow{7}{*}{$\begin{array}{l}2007- \\
2008\end{array}$} & Xigaze a & 3.7 & 1.3 & 22 & 2.7 & 4.4 & 29 & 12 & $6.4^{\mathrm{a}}$ \\
\hline & Xigaze b & 2.5 & 1.1 & 27 & 3.5 & 5.6 & 23 & 9.4 & 5.6 \\
\hline & Lhasa a & 3.7 & 1.2 & 57 & 1.9 & 5.2 & 25.9 & 6.6 & 3.6 \\
\hline & Lhasa b & 4.5 & 1.6 & 61.4 & 2.5 & 6.2 & 18.7 & 5.7 & 4.2 \\
\hline & Lhaze a & 1.4 & 0.7 & 21.7 & 0.9 & 1.2 & 10.5 & 2.6 & 2.6 \\
\hline & Lhaze b & 1.8 & 0.9 & 23.2 & 1.1 & 0.8 & 8.2 & 2.4 & 3.2 \\
\hline & RSD & $20 \%$ & $17 \%$ & $8 \%$ & $17 \%$ & $19 \%$ & $19 \%$ & $11 \%$ & $12 \%$ \\
\hline \multirow{11}{*}{$\begin{array}{l}2008- \\
2009\end{array}$} & Xigaze a & 6.55 & 2.14 & 10.04 & 0.95 & 1.40 & 2.19 & 3.35 & $0.67^{b}$ \\
\hline & Xigaze b & 6.72 & 3.03 & 14.07 & 1.00 & 1.27 & 2.90 & 3.22 & 0.59 \\
\hline & Namco a & 4.03 & 1.29 & 26.50 & 0.31 & 0.36 & 0.55 & 1.12 & 1.56 \\
\hline & Namco b & 3.19 & 1.35 & 26.40 & 0.15 & 0.17 & 2.10 & 0.87 & 0.35 \\
\hline & Lhaze a & 3.22 & 0.82 & 13.36 & 0.15 & 0.52 & 2.29 & 3.77 & 1.08 \\
\hline & Lhaze b & 5.48 & 1.32 & 23.97 & 0.52 & 0.51 & 2.23 & 1.22 & 1.09 \\
\hline & Lulang a & 2.78 & 0.84 & 7.94 & 0.91 & 0.42 & 6.36 & 5.23 & 0.29 \\
\hline & Lulang b & 3.01 & 0.91 & 9.04 & 0.77 & 1.19 & 2.96 & 5.99 & 0.90 \\
\hline & Everest a & 5.70 & 1.88 & 23.51 & 0.10 & 0.85 & 4.94 & 6.10 & 0.98 \\
\hline & Everest b & 4.31 & 1.59 & 24.98 & 0.15 & 0.25 & 6.72 & 3.35 & 0.33 \\
\hline & RSD & $16 \%$ & $15 \%$ & $16 \%$ & $34 \%$ & $41 \%$ & $36 \%$ & $29 \%$ & $48 \%$ \\
\hline \multirow{7}{*}{$\begin{array}{l}2009- \\
2010\end{array}$} & Namco a & 2.49 & 0.77 & 11.40 & 0.44 & 1.35 & 5.05 & 3.05 & $1.11^{\mathrm{b}}$ \\
\hline & Namco b & 3.78 & 0.92 & 20.57 & 0.88 & 1.57 & 17.86 & 9.69 & 1.59 \\
\hline & Lhasa a & 1.75 & 0.60 & 11.43 & 0.42 & 2.57 & 8.12 & 3.71 & 0.54 \\
\hline & Lhasa b & 1.72 & 0.46 & 8.54 & 0.41 & 2.72 & 7.37 & 3.21 & 0.37 \\
\hline & Everest a & 3.55 & 1.40 & 9.80 & 0.64 & 1.91 & 12.45 & 5.26 & 0.50 \\
\hline & Everest b & 4.75 & 1.09 & 18.95 & 0.54 & 2.06 & 9.96 & 4.50 & 0.47 \\
\hline & RSD\% & $17 \%$ & $16 \%$ & $35 \%$ & $20 \%$ & $7 \%$ & $34 \%$ & $32 \%$ & $19 \%$ \\
\hline \multirow{13}{*}{$\begin{array}{l}2010- \\
2011\end{array}$} & Xigaze a & 1.74 & 0.29 & 11.36 & 0.68 & 1.38 & 1.05 & 0.26 & $0.16^{\mathrm{b}}$ \\
\hline & Xigaze b & 1.50 & 0.19 & 11.92 & 0.40 & 1.45 & 0.78 & 0.22 & 0.16 \\
\hline & Namco a & 0.97 & 0.10 & 7.64 & 0.08 & 0.40 & 0.73 & 1.13 & 0.67 \\
\hline & Namco b & 1.29 & 0.24 & 9.45 & 0.13 & 0.19 & 0.93 & 0.12 & 0.16 \\
\hline & Lhasa a & 0.70 & 0.10 & 5.43 & 0.24 & 1.12 & 1.25 & 0.81 & 0.16 \\
\hline & Lhasa b & 0.75 & 0.11 & 5.10 & 0.24 & 0.87 & 1.07 & 0.38 & 0.16 \\
\hline & Lhaze a & 1.44 & 0.21 & 9.73 & 0.07 & 0.21 & 0.34 & 0.04 & 0.16 \\
\hline & Lhaze b & 1.03 & 0.13 & 10.06 & 0.05 & 0.09 & 0.39 & 0.03 & 0.16 \\
\hline & Lulang a & 1.83 & 0.21 & 6.71 & 0.26 & 0.59 & 2.19 & 0.92 & 0.16 \\
\hline & Lulang b & 0.98 & 0.11 & 8.06 & 0.22 & 0.83 & 1.94 & 0.95 & 0.16 \\
\hline & Everest a & 2.59 & 0.64 & 26.61 & 0.34 & 0.96 & 3.05 & 1.05 & 0.16 \\
\hline & Everest b & 2.01 & 0.46 & 17.86 & 0.22 & 0.51 & 1.74 & 0.68 & 0.16 \\
\hline & RSD & $20 \%$ & $32 \%$ & $11 \%$ & $23 \%$ & $33 \%$ & $18 \%$ & $38 \%$ & $12 \%$ \\
\hline \multirow{2}{*}{$\begin{array}{l}2011- \\
2012\end{array}$} & Xigaze a & 1.19 & 0.30 & 7.06 & 0.32 & 0.82 & 1.77 & 0.87 & $0.17^{\mathrm{b}}$ \\
\hline & Xigaze b & 1.02 & 0.24 & 6.36 & 0.29 & 0.75 & 1.34 & 0.67 & 0.14 \\
\hline
\end{tabular}




\begin{tabular}{|c|c|c|c|c|c|c|c|c|}
\hline Namco a & 1.40 & 0.35 & 11.22 & 0.09 & 0.25 & 2.09 & 0.81 & 0.18 \\
\hline Namco b & 1.23 & 0.32 & 8.93 & 0.09 & 0.18 & 1.86 & 0.68 & 0.17 \\
\hline Lhasa a & 0.62 & 0.15 & 3.57 & 0.25 & 1.27 & 2.20 & 1.32 & 0.19 \\
\hline Lhasa b & 0.57 & 0.14 & 3.72 & 0.36 & 1.51 & 3.00 & 1.99 & 0.22 \\
\hline Lhaze a & 1.08 & 0.22 & 5.47 & 0.10 & 0.41 & 1.21 & 1.08 & 0.21 \\
\hline Lhaze b & 1.22 & 0.19 & 6.07 & 0.11 & 0.33 & 1.81 & 0.98 & 0.16 \\
\hline Lulang a & 1.35 & 0.36 & 4.59 & 0.22 & 0.65 & 4.64 & 2.44 & 0.18 \\
\hline Lulang b & 2.16 & 0.49 & 5.84 & 0.36 & 1.12 & 6.03 & 3.86 & 0.25 \\
\hline Everest a & 1.81 & 0.49 & 11.17 & 0.17 & 0.53 & 3.94 & 1.68 & 0.22 \\
\hline Everest b & 1.80 & 0.63 & 12.78 & 0.27 & 0.97 & 4.28 & 2.89 & 0.29 \\
\hline RSD & $11 \%$ & $13 \%$ & $10 \%$ & $19 \%$ & $22 \%$ & $17 \%$ & $23 \%$ & $15 \%$ \\
\hline $\begin{array}{l}\text { Average RSD } \\
\text { for all duplicates }\end{array}$ & $17 \%$ & $20 \%$ & $15 \%$ & $16 \%$ & $24 \%$ & $24 \%$ & $27 \%$ & $21 \%$ \\
\hline
\end{tabular}

a: $\sum_{15}$ PCBs (data were from Wang et al., 2010);

b: $\sum_{6}$ PCBs.

Duplicate samples were deployed at 6 sites (Lhasa, Xigaze, Lhaze, Mt. Everest, Lulang and Namco), however some samples were destroyed during extraction and cleanup process. Thus data of 3 duplicates were reported for sampling periods from 2007 to 2008, and from 2009-2010; data of 5 duplicates were reported for sampling periods from 2008 to 2009. 
Table S4. Estimated Volumetric Air Concentrations (pg m-3) from the Tibetan Plateau Sites

\begin{tabular}{|c|c|c|c|c|c|c|c|c|c|c|}
\hline Sampling site & $\alpha-H C H$ & $\gamma-\mathrm{HCH}$ & HCB & o,p'-DDE & p,p'-DDE & o,p'-DDT & p,p'-DDT & PCB-28 & PCB-52 & $\sum_{6}$ PCBs \\
\hline \multicolumn{11}{|c|}{ 2007-2008 } \\
\hline Xigaze & 3.89 & 1.50 & 30.96 & 3.89 & 6.14 & 32.59 & 13.41 & 0.64 & 0.60 & 3.52 \\
\hline Nam co & 0.12 & 0.72 & 2.99 & 2.76 & 0.22 & 3.47 & 0.60 & 0.12 & 0.07 & 0.54 \\
\hline Lhasa & 4.84 & 1.65 & 69.84 & 2.60 & 6.72 & 26.31 & 7.31 & 0.64 & 0.18 & 1.77 \\
\hline Lhaze & 1.90 & 0.95 & 26.19 & 1.19 & 1.19 & 11.19 & 2.98 & 0.48 & 0.17 & 1.13 \\
\hline Lulang & 2.14 & 0.67 & 22.61 & 3.88 & 5.22 & 38.12 & 8.70 & 0.71 & 0.59 & 2.31 \\
\hline Everest & 4.70 & 5.64 & 22.99 & 0.10 & 0.31 & 3.24 & 0.52 & 2.19 & 0.10 & 2.38 \\
\hline Naqu & 9.70 & 2.52 & 36.39 & 2.26 & 1.06 & 20.32 & 5.45 & 0.37 & 0.33 & 1.82 \\
\hline Qamdo & 1.55 & 1.42 & 20.84 & 8.67 & 14.24 & 44.52 & 12.17 & 1.37 & 0.28 & 3.33 \\
\hline Saga & 5.11 & 1.67 & 21.07 & 3.34 & 5.95 & 29.73 & 9.39 & 0.42 & 0.31 & 1.26 \\
\hline Rawu & 17.65 & 20.13 & 12.70 & 1.18 & 4.41 & 41.87 & 7.75 & 2.56 & 0.18 & 2.83 \\
\hline Bomi & 9.10 & 5.04 & 85.00 & 2.80 & 5.88 & 16.10 & 4.20 & 1.25 & 0.67 & 3.93 \\
\hline GBJD & 8.27 & 2.52 & 27.70 & 4.08 & 8.15 & 33.69 & 7.79 & 0.48 & 0.28 & 2.23 \\
\hline Golmud & 4.93 & 1.70 & 75.17 & 2.55 & 2.04 & 2.38 & 0.85 & 1.07 & 0.46 & 1.67 \\
\hline Gar & 4.13 & 14.51 & 9.37 & 1.00 & 2.12 & 10.38 & 2.34 & 1.39 & 0.12 & 2.18 \\
\hline Chayu & I & I & I & / & I & I & I & I & I & / \\
\hline Muztagata & 1 & I & I & I & I & I & I & I & I & / \\
\hline \multicolumn{11}{|c|}{ 2008-2009 } \\
\hline Xigaze & 6.17 & 2.41 & 11.23 & 0.90 & 1.24 & 2.37 & 3.05 & 0.06 & 0.38 & 0.64 \\
\hline Nam co & 3.13 & 1.14 & 22.90 & 0.27 & 0.31 & 1.15 & 0.86 & 0.19 & 0.54 & 0.94 \\
\hline Lhasa & 4.51 & 2.65 & 27.20 & 0.14 & 0.85 & 4.41 & 2.22 & 0.20 & 0.49 & 0.86 \\
\hline Lhaze & 3.89 & 0.96 & 16.70 & 0.13 & 0.46 & 2.02 & 2.24 & 0.20 & 0.63 & 1.09 \\
\hline Lulang & 3.05 & 0.93 & 8.96 & 0.89 & 0.84 & 4.92 & 5.92 & 0.06 & 0.39 & 0.66 \\
\hline Everest & 4.75 & 1.64 & 22.99 & 0.14 & 0.52 & 5.53 & 4.48 & 0.10 & 0.45 & 0.75 \\
\hline
\end{tabular}




\begin{tabular}{ccccccccccc} 
Naqu & 9.49 & 2.27 & 10.82 & 0.13 & 0.15 & 1.02 & 1.83 & 0.05 & 0.34 & 0.58 \\
Qamdo & 2.25 & 0.90 & 11.20 & 0.15 & 1.27 & 0.40 & 2.17 & 0.21 & 0.57 & 1.05 \\
Saga & 3.38 & 0.96 & 10.55 & 0.11 & 0.70 & 2.28 & 2.46 & 0.14 & 0.36 & 0.66 \\
Rawu & 1.81 & 0.37 & 6.93 & 0.16 & 0.19 & 0.77 & 1.73 & 0.07 & 0.14 & 0.42 \\
Bomi & 16.58 & 4.63 & 31.92 & 0.71 & 4.90 & 11.43 & 25.95 & 0.18 & 0.58 & 1.03 \\
GBJD & $/$ & $/$ & $/$ & $/$ & $/$ & $/$ & $/$ & $/$ & $/$ & $/$ \\
Golmud & 6.15 & 2.92 & 43.03 & 0.19 & 0.22 & 0.13 & 0.13 & 0.32 & 0.89 & 1.45 \\
Gar & 5.72 & 1.73 & 26.31 & 0.57 & 0.85 & 0.09 & 0.93 & 0.24 & 0.65 & 1.07 \\
Chayu & 5.35 & 1.35 & 25.81 & 0.24 & 1.75 & 9.57 & 24.91 & 0.10 & 0.48 & 0.88 \\
Muatagata & $/$ & $/$ & $/$ & $/$ & $/$ & $/$ & $/$ & $/$ & $/$ & $/$ \\
\hline & & & & & $\mathbf{2 0 0 9 - 2 0 1 0}$ & & & & & 0.30 \\
\hline Xigaze & 1.95 & 0.61 & 9.46 & 3.04 & 4.52 & 8.63 & 3.70 & 0.07 & 0.02 & 0.30 \\
Nam co & 2.70 & 0.72 & 13.73 & 0.57 & 1.25 & 9.85 & 5.47 & 0.06 & 0.10 & 1.17 \\
Lhasa & 1.80 & 0.55 & 10.41 & 0.44 & 2.75 & 8.07 & 3.61 & 0.07 & 0.05 & 0.54 \\
Lhaze & 3.55 & 1.22 & 10.54 & 0.09 & 1.43 & 4.15 & 2.93 & 0.06 & 0.05 & 0.37 \\
Lulang & 3.40 & 0.82 & 9.33 & 0.38 & 2.27 & 12.88 & 6.38 & 0.08 & 0.07 & 0.45 \\
Everest & 3.70 & 1.10 & 12.80 & 0.53 & 1.76 & 9.98 & 4.35 & 0.06 & 0.08 & 0.42 \\
Naqu & 9.94 & 2.25 & 14.14 & 0.09 & 0.99 & 3.99 & 1.80 & 0.06 & 0.06 & 0.30 \\
Qamdo & 3.14 & 1.24 & 12.18 & 0.24 & 1.96 & 3.41 & 2.81 & 0.17 & 0.17 & 0.61 \\
Saga & 2.23 & 0.57 & 8.46 & 0.09 & 1.67 & 4.16 & 4.18 & 0.06 & 0.04 & 0.28 \\
Rawu & 3.16 & 0.79 & 12.75 & 0.18 & 1.09 & 5.84 & 2.98 & 0.06 & 0.05 & 0.24 \\
Bomi & 8.32 & 2.60 & 8.79 & 1.48 & 18.09 & 31.85 & 26.09 & 0.30 & 0.02 & 0.82 \\
GBJD & $/$ & $/$ & $/$ & $/$ & $/$ & $/$ & $/$ & $/$ & $/$ & $/$ \\
Golmud & 7.04 & 1.86 & 38.90 & 0.23 & 1.42 & 6.01 & 2.03 & 0.28 & 0.33 & 1.11 \\
Gar & 1.31 & 0.88 & 13.57 & 0.10 & 1.04 & 1.53 & 2.01 & 0.07 & 0.08 & 0.59 \\
Chayu & 7.87 & 2.15 & 31.41 & 1.68 & 8.75 & 37.33 & 20.20 & 0.21 & 0.16 & 0.83 \\
Muztaga & 2.33 & 0.47 & 20.72 & 0.13 & 1.27 & 1.29 & 1.65 & 0.09 & 0.03 & 0.39
\end{tabular}




\begin{tabular}{|c|c|c|c|c|c|c|c|c|c|c|}
\hline \multicolumn{11}{|c|}{2010 - 2011} \\
\hline Xigaze & 1.72 & 0.25 & 12.37 & 0.57 & 1.50 & 0.98 & 0.19 & 0.13 & 0.04 & 0.33 \\
\hline Nam co & 1.10 & 0.17 & 8.30 & 0.10 & 0.19 & 0.81 & 0.61 & 0.34 & 0.04 & 0.52 \\
\hline Lhasa & 0.80 & 0.12 & 5.79 & 0.26 & 1.09 & 1.27 & 0.65 & 0.13 & 0.04 & 0.34 \\
\hline Lhaze & 1.27 & 0.18 & 10.23 & 0.10 & 0.21 & 0.37 & 0.19 & 0.12 & 0.04 & 0.32 \\
\hline Lulang & 1.73 & 0.20 & 9.09 & 0.30 & 0.87 & 2.53 & 1.14 & 0.15 & 0.05 & 0.38 \\
\hline Everest & 2.20 & 0.53 & 21.26 & 0.27 & 0.70 & 2.29 & 0.83 & 0.11 & 0.08 & 0.33 \\
\hline Naqu & 6.08 & 1.08 & 12.91 & 0.10 & 0.20 & 0.71 & 0.18 & 0.12 & 0.04 & 0.31 \\
\hline Qamdo & 1.63 & 0.26 & 10.85 & 0.12 & 0.49 & 0.53 & 0.22 & 0.14 & 0.05 & 0.37 \\
\hline Saga & 3.10 & 0.49 & 14.75 & 0.09 & 0.61 & 0.86 & 0.96 & 0.11 & 0.04 & 0.28 \\
\hline Rawu & 1.55 & 0.33 & 7.19 & 0.21 & 0.56 & 1.27 & 0.41 & 0.38 & 0.04 & 0.58 \\
\hline Bomi & 5.21 & 0.79 & 6.87 & 0.35 & 5.62 & 2.67 & 1.52 & 0.16 & 0.05 & 0.42 \\
\hline GBJD & 4.23 & 0.99 & 10.65 & 0.29 & 1.27 & 3.86 & 1.77 & 0.14 & 0.05 & 0.37 \\
\hline Golmud & 1.97 & 0.26 & 30.61 & 0.15 & 0.31 & 0.51 & 0.28 & 0.18 & 0.11 & 0.52 \\
\hline Gar & 0.70 & 0.11 & 9.33 & 0.10 & 0.21 & 0.16 & 0.19 & 0.12 & 0.04 & 0.32 \\
\hline Chayu & 1.88 & 0.23 & 12.94 & 0.95 & 7.09 & 11.56 & 7.69 & 0.17 & 0.06 & 0.44 \\
\hline Muztaga & 2.69 & 0.28 & 31.78 & 0.13 & 1.13 & 0.19 & 0.23 & 0.15 & 0.11 & 0.45 \\
\hline \multicolumn{11}{|c|}{$2011-2012$} \\
\hline Xigaze & 1.12 & 0.28 & 6.84 & 0.32 & 0.79 & 1.59 & 0.78 & 0.03 & 0.03 & 0.14 \\
\hline Nam co & 1.21 & 0.31 & 9.31 & 0.08 & 0.20 & 1.83 & 0.69 & 0.03 & 0.03 & 0.14 \\
\hline Lhasa & 0.62 & 0.14 & 3.74 & 0.32 & 1.43 & 2.67 & 1.69 & 0.03 & 0.02 & 0.24 \\
\hline Lhaze & 1.10 & 0.20 & 5.52 & 0.10 & 0.35 & 1.44 & 0.99 & 0.03 & 0.02 & 0.14 \\
\hline Lulang & 2.03 & 0.49 & 6.06 & 0.34 & 1.03 & 6.19 & 3.66 & 0.03 & 0.05 & 0.19 \\
\hline Everest & 1.72 & 0.53 & 11.42 & 0.21 & 0.72 & 3.92 & 2.18 & 0.03 & 0.05 & 0.25 \\
\hline Naqu & 6.15 & 1.38 & 13.98 & 0.12 & 0.37 & 1.64 & 1.50 & 0.06 & 0.06 & 0.21 \\
\hline Qamdo & 0.96 & 0.29 & 4.74 & 0.14 & 0.74 & 1.12 & 0.92 & 0.03 & 0.03 & 0.27 \\
\hline Saga & 1.05 & 0.32 & 5.50 & 0.08 & 0.25 & 0.83 & 0.45 & 0.03 & 0.03 & 0.12 \\
\hline
\end{tabular}




\begin{tabular}{ccccccccccc} 
Rawu & 1.06 & 0.28 & 4.35 & 0.19 & 0.51 & 2.74 & 1.56 & 0.03 & 0.03 & 0.15 \\
Bomi & 1.46 & 0.36 & 3.57 & 0.52 & 3.82 & 7.08 & 4.90 & 0.04 & 0.01 & 0.50 \\
GBJD & 2.47 & 0.54 & 8.31 & 0.30 & 1.29 & 5.77 & 25.62 & 0.07 & 0.05 & 0.30 \\
Golmud & 2.58 & 0.96 & 24.90 & 0.07 & 0.64 & 0.54 & 0.73 & 0.18 & 0.10 & 0.66 \\
Gar & 0.79 & 0.20 & 6.58 & 0.04 & 0.18 & 0.42 & 0.34 & 0.03 & 0.03 & 0.14 \\
Chayu & 2.21 & 0.44 & 8.66 & 0.58 & 3.31 & 11.06 & 9.01 & 0.04 & 0.04 & 0.31 \\
Muztagata & 2.85 & 0.42 & 27.85 & 0.03 & 1.52 & 1.01 & 1.65 & 0.04 & 0.03 & 0.30 \\
\hline
\end{tabular}




\section{Text S3 The comparison between AAS and PAS}

To test if the $\mathrm{R}$ used in this study is rational, a direct concentration comparison between PAS and AAS is imperative owing to the relatively accurate air volume of AAS. There are one site, Lulang, where two kinds of samplers were both deployed, thus provides the possibility for the comparison. Data of AAS were collected from the previous study (Sheng et al., 2013). The sampling period of AAS was from September 2008 to August 2011, which overlapped with PAS sampling. AAS data were then averaged and compared with data obtained from PAS. The results are presented below.

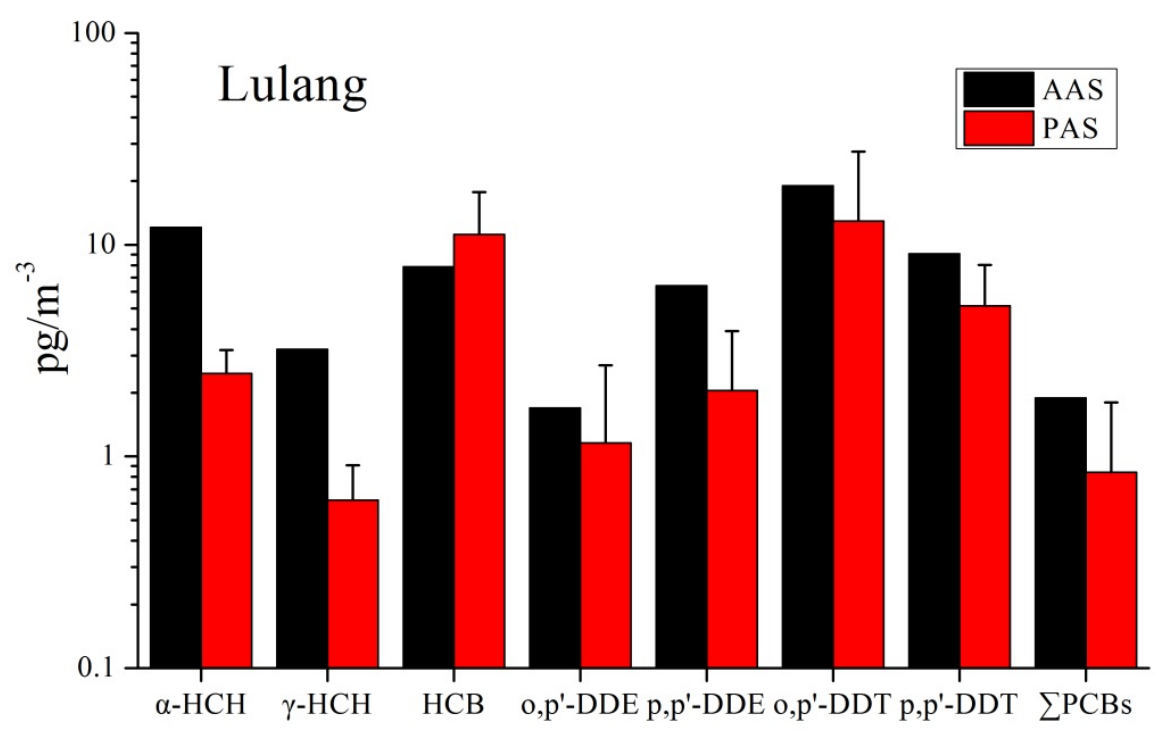

The DDTs derived from PAS had no much difference from the corresponding AAS concentrations; whereas the low molecular weight OCPs, such as $\alpha-\mathrm{HCH}$ and $\gamma-\mathrm{HCH}$, showed some discrepancy (Figure above). This may be caused by different air masses being sampled and different adsorption characteristics for two kinds of samplers. Under these restrictions, the concentration variability within a factor of 2 or 3 is deemed to be acceptable (Gouin et al., 2005). Therefore, the differences in the present PAS/AAS comparison (Figure above) were acceptable, which demonstrate that the $\mathrm{R}$ obtained in our study was reasonable. 
Table S5 Average air concentrations (ng/sample) for each sampling site during the 5 sampling years

\begin{tabular}{|c|c|c|c|c|c|c|c|c|c|c|}
\hline $\begin{array}{c}\text { Sampling } \\
\text { site }\end{array}$ & $\alpha-\mathbf{H C H}$ & $\gamma-\mathbf{H C H}$ & НСB & o,p'-DDE & p,p'-DDE & o,p'-DDT & p,p'-DDT & $\begin{array}{c}\text { PCB- } \\
28 \\
\end{array}$ & $\begin{array}{c}\text { PCB- } \\
52 \\
\end{array}$ & $\sum 6 \mathrm{PCBs}$ \\
\hline Xigaze & 2.9 & 1.0 & 13.0 & 1.6 & 2.6 & 8.0 & 3.8 & 0.2 & 0.2 & 0.9 \\
\hline Nam co & 1.9 & 0.7 & 12.7 & 0.7 & 0.5 & 3.7 & 1.8 & 0.2 & 0.2 & 0.7 \\
\hline Lhasa & 2.4 & 1.0 & 21.0 & 0.7 & 2.3 & 7.7 & 2.9 & 0.2 & 0.2 & 0.7 \\
\hline Lhaze & 2.4 & 0.7 & 13.6 & 0.3 & 0.7 & 3.6 & 1.9 & 0.2 & 0.2 & 0.6 \\
\hline Lulang & 2.2 & 0.5 & 9.3 & 0.9 & 1.7 & 10.5 & 4.4 & 0.2 & 0.2 & 0.6 \\
\hline Everest & 3.6 & 1.9 & 19.0 & 0.3 & 0.9 & 5.3 & 2.7 & 0.5 & 0.2 & 0.8 \\
\hline Naqu & 8.3 & 1.9 & 16.6 & 0.4 & 0.5 & 4.7 & 2.0 & 0.1 & 0.2 & 0.6 \\
\hline Qamdo & 1.7 & 0.7 & 10.4 & 1.5 & 3.0 & 7.9 & 3.0 & 0.3 & 0.2 & 0.9 \\
\hline Saga & 3.3 & 0.9 & 13.4 & 0.7 & 1.9 & 7.7 & 3.7 & 0.2 & 0.2 & 0.6 \\
\hline Rawu & 4.9 & 4.1 & 9.1 & 0.4 & 1.3 & 10.2 & 2.9 & 0.6 & 0.1 & 0.8 \\
\hline Bomi & 6.5 & 2.1 & 20.6 & 0.9 & 6.1 & 11.0 & 10.3 & 0.3 & 0.2 & 1.0 \\
\hline GBJD & 4.2 & 1.1 & 13.2 & 1.3 & 3.0 & 12.2 & 10.2 & 0.2 & 0.1 & 0.8 \\
\hline Golmud & 3.4 & 1.2 & 30.0 & 0.4 & 0.7 & 1.5 & 0.6 & 0.3 & 0.3 & 0.8 \\
\hline Gar & 2.6 & 3.2 & 13.5 & 0.4 & 0.9 & 2.3 & 1.1 & 0.3 & 0.2 & 0.7 \\
\hline Chayu & 3.2 & 0.8 & 14.5 & 0.7 & 4.0 & 13.4 & 11.1 & 0.1 & 0.1 & 0.4 \\
\hline Muztagata & 2.1 & 0.3 & 21.2 & 0.1 & 1.0 & 0.7 & 0.9 & 0.1 & 0.0 & 0.3 \\
\hline
\end{tabular}


Table S6 Average volumetric concentrations $\left(\mathrm{pg} / \mathrm{m}^{3}\right)$ for each sampling site during the 5 sampling years

\begin{tabular}{ccccccccccc}
\hline $\begin{array}{c}\text { sampling } \\
\text { site }\end{array}$ & \multirow{2}{\alpha}{-HCH } & $\boldsymbol{\gamma}$-HCH & \multirow{2}{*}{ HCB } & $\begin{array}{c}\mathbf{0 , p}- \\
\mathbf{D D E}\end{array}$ & $\begin{array}{c}\text { p,p'- } \\
\text { DDE }\end{array}$ & $\begin{array}{c}\mathbf{0 , p}- \\
\text { DDT }\end{array}$ & $\begin{array}{c}\text { p,p'- } \\
\text { DDT }\end{array}$ & $\begin{array}{c}\text { PCB- } \\
\mathbf{2 8}\end{array}$ & $\begin{array}{c}\text { PCB- } \\
\mathbf{5 2}\end{array}$ & $\sum \mathbf{6}$ PCBs \\
\hline Xigaze & 3.0 & 1.0 & 14.2 & 1.7 & 2.8 & 9.2 & 4.2 & 0.2 & 0.2 & 1.0 \\
Nam co & 1.7 & 0.6 & 11.4 & 0.8 & 0.4 & 3.4 & 1.6 & 0.1 & 0.2 & 0.7 \\
Lhasa & 2.5 & 1.0 & 23.4 & 0.8 & 2.6 & 8.5 & 3.1 & 0.2 & 0.2 & 0.8 \\
Lhaze & 2.3 & 0.7 & 13.8 & 0.3 & 0.7 & 3.8 & 1.9 & 0.2 & 0.2 & 0.6 \\
Lulang & 2.5 & 0.6 & 11.2 & 1.2 & 2.0 & 12.9 & 5.2 & 0.2 & 0.2 & 0.8 \\
Everest & 3.4 & 1.9 & 18.3 & 0.2 & 0.8 & 5.0 & 2.5 & 0.5 & 0.2 & 0.8 \\
Naqu & 8.3 & 1.9 & 17.7 & 0.5 & 0.6 & 5.5 & 2.2 & 0.1 & 0.2 & 0.6 \\
Qamdo & 1.9 & 0.8 & 12.0 & 1.9 & 3.7 & 10.0 & 3.7 & 0.4 & 0.2 & 1.1 \\
Saga & 3.0 & 0.8 & 12.1 & 0.7 & 1.8 & 7.6 & 3.5 & 0.2 & 0.2 & 0.5 \\
Rawu & 5.0 & 4.4 & 8.8 & 0.4 & 1.4 & 10.5 & 2.9 & 0.6 & 0.1 & 0.8 \\
Bomi & 8.1 & 2.7 & 27.2 & 1.2 & 7.7 & 13.8 & 12.5 & 0.4 & 0.3 & 1.3 \\
GBJD & 5.0 & 1.3 & 15.6 & 1.6 & 3.6 & 14.4 & 11.7 & 0.2 & 0.1 & 1.0 \\
Golmud & 4.5 & 1.5 & 42.5 & 0.6 & 0.9 & 1.9 & 0.8 & 0.4 & 0.4 & 1.1 \\
Gar & 2.5 & 3.5 & 13.0 & 0.4 & 0.9 & 2.5 & 1.2 & 0.4 & 0.2 & 0.9 \\
Chayu & 4.3 & 1.0 & 19.7 & 0.9 & 5.2 & 17.4 & 15.5 & 0.1 & 0.2 & 0.6 \\
Muztagata & 2.6 & 0.4 & 26.8 & 0.1 & 1.3 & 0.8 & 1.2 & 0.1 & 0.1 & 0.4 \\
\hline
\end{tabular}


Text S4 A comparison between concentrations of the current study and the previous Global Atmospheric Passive Sampling (GAPS) study

To further identify the POPs contamination level on the global scale, a comparison was performed between the current study and the previous Global Atmospheric Passive Sampling (GAPS) study which also relied on the XAD-PAS (Shunthirasingham et al., 2010). The levels of HCB in the Tibetan atmosphere ranged from 2.5 to $60.7 \mathrm{ng} / \mathrm{sample}$ (Table S2), which is higher than the background areas of Asia (4.6-27.4 ng/sample) and South America (8.9-17.8 ng/sample), but similar to those in Europe (12.6-78.1 ng/sample) (Shunthirasingham et al., 2010). The level of $\alpha-\mathrm{HCH}(0.1-16.4 \mathrm{ng} / \mathrm{sample})$ and $\gamma-\mathrm{HCH}(0.1-18.7 \mathrm{ng} / \mathrm{sample})$ is comparable to the remote stations in North America (Below detection limit-15.3 ng/sample) and Africa (1.4-18.7 ng/sample), respectively (Shunthirasingham et al., 2010). Regarding the PCBs, its value ranged from 0.1 to $2.8 \mathrm{ng} /$ sample in this study, which is much lower than those of Arctic Region (1.7-14 ng/sample) (Shunthirasingham et al., 2010). These comparisons indicate that POPs concentrations in the atmosphere of TP are akin to those observed in the background sites of the world, indicating the remoteness of TP. However, it is worth noting that the DDTs levels of TP (0.1-38.9 ng/sample for o.p'-DDT and 0.9-22.7 ng/sample for p-p'-DDT, Table S2) were considerably higher than other remote regions (mostly not detected) (Shunthirasingham et al., 2010), which may suggest closer proximity of the TP to the DDT source regions. 
Table S7. $P$ values of randomized block ANOVA test for the concentrations of DDTs, HCHs, HCB and PCBs in the different sampling sites. $(p<0.05$ indicates that the concentrations significantly differ between two sites.)

\begin{tabular}{|c|c|c|c|c|c|}
\hline \multicolumn{2}{|c|}{ Sampling site } & DDTs & HCHs & HCB & PCBs \\
\hline \multirow[t]{15}{*}{ Xigaze } & Nam co & 0.306 & 0.621 & 0.764 & 0.560 \\
\hline & Lhasa & 0.788 & 0.898 & 0.312 & 0.671 \\
\hline & Lhaze & 0.326 & 0.787 & 0.970 & 0.499 \\
\hline & Lulang & 0.777 & 0.798 & 0.744 & 0.735 \\
\hline & Everest & 0.407 & 0.703 & 0.650 & 0.773 \\
\hline & Naqu & 0.421 & 0.078 & 0.702 & 0.539 \\
\hline & Qamdo & 0.916 & 0.719 & 0.808 & 0.801 \\
\hline & Saga & 0.700 & 0.952 & 0.817 & 0.403 \\
\hline & Rawu & 0.799 & 0.120 & 0.553 & 0.798 \\
\hline & Bomi & 0.139 & 0.053 & 0.154 & 0.525 \\
\hline & GBJD & 0.319 & 0.556 & 0.895 & 0.976 \\
\hline & Golmud & 0.233 & 0.547 & 0.003 & 0.863 \\
\hline & Gar & 0.255 & 0.558 & 0.900 & 0.666 \\
\hline & Chayu & 0.090 & 0.706 & 0.566 & 0.530 \\
\hline & Muztagata & 0.271 & 0.808 & 0.232 & 0.347 \\
\hline \multirow[t]{14}{*}{ Nam co } & Lhasa & 0.449 & 0.714 & 0.191 & 0.874 \\
\hline & Lhaze & 0.966 & 0.822 & 0.792 & 0.925 \\
\hline & Lulang & 0.193 & 0.811 & 0.979 & 0.807 \\
\hline & Everest & 0.844 & 0.383 & 0.452 & 0.768 \\
\hline & Naqu & 0.826 & 0.026 & 0.495 & 0.974 \\
\hline & Qamdo & 0.260 & 0.893 & 0.955 & 0.405 \\
\hline & Saga & 0.521 & 0.664 & 0.946 & 0.798 \\
\hline & Rawu & 0.441 & 0.043 & 0.769 & 0.743 \\
\hline & Bomi & 0.014 & 0.016 & 0.086 & 0.225 \\
\hline & GBJD & 0.062 & 0.311 & 0.695 & 0.635 \\
\hline & Golmud & 0.863 & 0.275 & 0.001 & 0.451 \\
\hline & Gar & 0.907 & 0.282 & 0.861 & 0.880 \\
\hline & Chayu & 0.009 & 0.400 & 0.392 & 0.936 \\
\hline & Muztagata & 0.829 & 0.852 & 0.147 & 0.660 \\
\hline \multirow[t]{11}{*}{ Lhasa } & Lhaze & 0.475 & 0.887 & 0.294 & 0.801 \\
\hline & Lulang & 0.581 & 0.898 & 0.183 & 0.931 \\
\hline & Everest & 0.575 & 0.611 & 0.574 & 0.891 \\
\hline & Naqu & 0.591 & 0.060 & 0.527 & 0.849 \\
\hline & Qamdo & 0.708 & 0.816 & 0.211 & 0.499 \\
\hline & Saga & 0.908 & 0.946 & 0.215 & 0.679 \\
\hline & Rawu & 0.988 & 0.094 & 0.111 & 0.866 \\
\hline & Bomi & 0.082 & 0.039 & 0.673 & 0.290 \\
\hline & GBJD & 0.220 & 0.485 & 0.455 & 0.736 \\
\hline & Golmud & 0.354 & 0.466 & 0.039 & 0.551 \\
\hline & Gar & 0.383 & 0.476 & 0.256 & 0.994 \\
\hline
\end{tabular}




\begin{tabular}{|c|c|c|c|c|c|}
\hline & Chayu & 0.052 & 0.619 & 0.702 & 0.819 \\
\hline & Muztagata & 0.384 & 0.895 & 0.747 & 0.565 \\
\hline \multirow[t]{12}{*}{ Lhaze } & Lulang & 0.208 & 0.989 & 0.772 & 0.735 \\
\hline & Everest & 0.877 & 0.516 & 0.624 & 0.698 \\
\hline & Naqu & 0.859 & 0.044 & 0.675 & 0.951 \\
\hline & Qamdo & 0.278 & 0.928 & 0.836 & 0.355 \\
\hline & Saga & 0.549 & 0.834 & 0.845 & 0.871 \\
\hline & Rawu & 0.466 & 0.070 & 0.578 & 0.674 \\
\hline & Bomi & 0.016 & 0.028 & 0.144 & 0.192 \\
\hline & GBJD & 0.068 & 0.412 & 0.087 & 0.579 \\
\hline & Golmud & 0.830 & 0.384 & 0.002 & 0.397 \\
\hline & Gar & 0.873 & 0.394 & 0.929 & 0.807 \\
\hline & Chayu & 0.010 & 0.529 & 0.543 & 0.993 \\
\hline & Muztagata & 0.801 & 0.993 & 0.220 & 0.720 \\
\hline \multirow[t]{11}{*}{ Lulang } & Everest & 0.268 & 0.525 & 0.436 & 0.960 \\
\hline & Naqu & 0.278 & 0.045 & 0.479 & 0.782 \\
\hline & Qamdo & 0.859 & 0.917 & 0.934 & 0.555 \\
\hline & Saga & 0.505 & 0.845 & 0.925 & 0.617 \\
\hline & Rawu & 0.591 & 0.072 & 0.789 & 0.934 \\
\hline & Bomi & 0.228 & 0.029 & 0.081 & 0.331 \\
\hline & GBJD & 0.451 & 0.419 & 0.679 & 0.793 \\
\hline & Golmud & 0.142 & 0.392 & 0.001 & 0.610 \\
\hline & Gar & 0.157 & 0.401 & 0.841 & 0.925 \\
\hline & Chayu & 0.151 & 0.537 & 0.379 & 0.756 \\
\hline & Muztagata & 0.180 & 0.983 & 0.141 & 0.515 \\
\hline \multirow[t]{10}{*}{ Everest } & Naqu & 0.981 & 0.164 & 0.943 & 0.743 \\
\hline & Qamdo & 0.351 & 0.460 & 0.486 & 0.590 \\
\hline & Saga & 0.656 & 0.660 & 0.494 & 0.582 \\
\hline & Rawu & 0.565 & 0.238 & 0.297 & 0.974 \\
\hline & Bomi & 0.023 & 0.116 & 0.327 & 0.356 \\
\hline & GBJD & 0.089 & 0.795 & 0.794 & 0.826 \\
\hline & Golmud & 0.712 & 0.824 & 0.009 & 0.645 \\
\hline & Gar & 0.754 & 0.838 & 0.563 & 0.885 \\
\hline & Chayu & 0.015 & 0.986 & 0.883 & 0.720 \\
\hline & Muztagata & 0.700 & 0.568 & 0.419 & 0.488 \\
\hline \multirow[t]{9}{*}{ Naqu } & Qamdo & 0.363 & 0.035 & 0.532 & 0.387 \\
\hline & Saga & 0.673 & 0.069 & 0.539 & 0.823 \\
\hline & Rawu & 0.581 & 0.830 & 0.331 & 0.719 \\
\hline & Bomi & 0.024 & 0.852 & 0.293 & 0.213 \\
\hline & GBJD & 0.093 & 0.341 & 0.842 & 0.615 \\
\hline & Golmud & 0.695 & 0.241 & 0.008 & 0.432 \\
\hline & Gar & 0.736 & 0.234 & 0.611 & 0.854 \\
\hline & Chayu & 0.016 & 0.195 & 0.831 & 0.961 \\
\hline & Muztagata & 0.685 & 0.078 & 0.385 & 0.681 \\
\hline \multirow[t]{2}{*}{ Qamdo } & Saga & 0.624 & 0.764 & 0.991 & 0.278 \\
\hline & Rawu & 0.719 & 0.058 & 0.726 & 0.612 \\
\hline
\end{tabular}




\begin{tabular}{|c|c|c|c|c|c|}
\hline & Bomi & 0.168 & 0.023 & 0.096 & 0.700 \\
\hline & GBJD & 0.365 & 0.369 & 0.732 & 0.804 \\
\hline & Golmud & 0.195 & 0.337 & 0.001 & 0.937 \\
\hline & Gar & 0.214 & 0.346 & 0.906 & 0.495 \\
\hline & Chayu & 0.110 & 0.475 & 0.422 & 0.387 \\
\hline & Muztagata & 0.234 & 0.945 & 0.161 & 0.247 \\
\hline \multirow[t]{7}{*}{ Saga } & Rawu & 0.897 & 0.107 & 0.718 & 0.560 \\
\hline & Bomi & 0.064 & 0.046 & 0.099 & 0.143 \\
\hline & GBJD & 0.185 & 0.522 & 0.739 & 0.487 \\
\hline & Golmud & 0.416 & 0.508 & 0.001 & 0.314 \\
\hline & Gar & 0.449 & 0.519 & 0.915 & 0.684 \\
\hline & Chayu & 0.041 & 0.665 & 0.429 & 0.872 \\
\hline & Muztagata & 0.441 & 0.849 & 0.164 & 0.827 \\
\hline \multirow[t]{6}{*}{ Rawu } & Bomi & 0.084 & 0.689 & 0.046 & 0.373 \\
\hline & GBJD & 0.225 & 0.443 & 0.519 & 0.848 \\
\hline & Golmud & 0.346 & 0.336 & 0.000 & 0.669 \\
\hline & Gar & 0.375 & 0.328 & 0.640 & 0.860 \\
\hline & Chayu & 0.054 & 0.273 & 0.259 & 0.698 \\
\hline & Muztagata & 0.378 & 0.113 & 0.090 & 0.470 \\
\hline \multirow[t]{5}{*}{ Bomi } & GBJD & 0.769 & 0.267 & 0.268 & 0.561 \\
\hline & Golmud & 0.009 & 0.175 & 0.096 & 0.643 \\
\hline & Gar & 0.010 & 0.170 & 0.121 & 0.287 \\
\hline & Chayu & 0.759 & 0.143 & 0.436 & 0.221 \\
\hline & Muztagata & 0.019 & 0.055 & 0.966 & 0.138 \\
\hline \multirow[t]{4}{*}{ GBJD } & Golmud & 0.045 & 0.947 & 0.012 & 0.857 \\
\hline & Gar & 0.050 & 0.935 & 0.810 & 0.731 \\
\hline & Chayu & 0.582 & 0.816 & 0.705 & 0.600 \\
\hline & Muztagata & 0.063 & 0.458 & 0.340 & 0.415 \\
\hline \multirow[t]{3}{*}{ Golmud } & Gar & 0.955 & 0.986 & 0.002 & 0.546 \\
\hline & Chayu & 0.006 & 0.848 & 0.021 & 0.429 \\
\hline & Muztagata & 0.947 & 0.445 & 0.137 & 0.276 \\
\hline \multirow[t]{2}{*}{ Gar } & Chayu & 0.007 & 0.861 & 0.489 & 0.824 \\
\hline & Muztagata & 0.909 & 0.454 & 0.193 & 0.569 \\
\hline Chayu & Muztagata & 0.013 & 0.574 & 0.519 & 0.726 \\
\hline
\end{tabular}




\section{Text S5 Calculation equations for fugacity, fugacity ratio and fugacity fluxes}

Air-soil exchange directions and fluxes are very important for understanding the global cycling of POPs, which will help to understand whether the chemical can be evaporated from soil and how much the exchange fluxes will be.

Air fugacity of the chemicals can be represented as:

$\mathrm{f}_{\mathrm{a}}=\mathrm{C}_{\mathrm{a}} \mathrm{RT}(1)$.

Soil fugacity of the chemicals can be presented as:

$f_{s}=C_{s} \rho_{s} R T / 0.41 \varphi_{o m} K_{o a}(2)$

where $C_{s}$ is the soil concentration (ng/g), $\rho_{s}$ is the density of soil solids (g/m3), $\varphi_{\text {om }}$ is the fraction of organic matter in the soil on a dry soil basis, $K_{o a}$ is the octanol-air partition coefficient of the compound, $\mathrm{R}$ is the gas constant (8.31 $\left.\mathrm{pa} \mathrm{m}^{3} / \mathrm{mol} / \mathrm{K}\right)$ and $\mathrm{T}$ is the average air temperature (K).

Fugacity fraction(ff) could be calculated by $f f=f_{s} /\left(f_{s}+f_{a}\right)$ (3)

Ideally, the $\mathrm{ff}=0.5$ indicates that soil fugacity and air fugacity is same and the exchange reach equilibrium. However, assuming an error of approximately $20 \%$ in $\mathrm{K}_{\mathrm{OA}}$ and $35 \%$ in analysis results in a propagated error of approximately $40 \%$ in the $\mathrm{ff}$, a fugacity fraction in the range $0.5 \pm$ 0.2 may not represent a significant departure from equilibrium. Therefore, the criterion range representing the equilibrium can vary from 0.3 to 0.7 (Harner et al., 2001 and Li et al., 2009).

Flux quantification $(F)$ can provide detailed data on how much POP has been volatilizing from air or depositing into soil.

$F=\left(f_{a}-f_{s}\right) /\left(R T / K_{13}+L_{3} *\left(R T / B_{1}+H / B_{2}\right)\right)$,

where $K_{13}, L_{3}, B_{1}$ and $B_{2}$ are air-soil mass transfer coefficient (m/h), diffusion path length in soil $(\mathrm{m})$, molecular diffusivities in air and water $\left(\mathrm{m}^{2} / \mathrm{h}\right)$, respectively $\left(\mathrm{K}_{13}=3.75 \mathrm{~m} / \mathrm{h}, \mathrm{L}_{3}=0.1 \mathrm{~m}\right.$, $\mathrm{B}_{1}=0.0179 \mathrm{~m}^{2} / \mathrm{h}, \mathrm{B}_{2}=1.79 \times 10^{-6} \mathrm{~m}^{2} / \mathrm{h}$, these values were cited from McLachlan et al.( 2002). $\mathrm{H}$ is Henry constant (Pa m3/mol). 
Table S8 The soil-air equilibrium status ( $f f$ ) of Tibetan Plateau sites.

\begin{tabular}{|c|c|c|c|c|c|c|c|c|c|c|c|c|c|}
\hline \multicolumn{14}{|c|}{ 2007-2008 } \\
\hline & GBJD & Lulang & Bomi & Rawu & Qamdo & Lhasa & Namco & Lhaze & Xigaze & Everest & Saga & Naqu & Gar \\
\hline НСВ & 0.66 & 0.58 & 0.59 & 0.70 & 0.86 & 0.74 & 0.77 & 0.91 & 0.60 & 0.81 & 0.79 & 0.65 & 0.94 \\
\hline PCB-28 & 0.58 & 0.52 & 0.68 & 0.06 & 0.82 & 0.99 & 0.90 & 1.00 & 0.97 & 0.85 & 0.98 & 0.96 & 0.97 \\
\hline PCB-52 & 0.94 & 0.75 & 0.95 & 0.18 & 0.89 & 0.99 & 0.44 & 0.93 & - & - & 0.59 & 0.38 & - \\
\hline & 0.29 & 0.87 & 0.81 & 0.31 & 0.97 & 0.88 & 0.98 & 0.99 & 0.64 & 0.86 & 0.59 & 0.58 & 0.90 \\
\hline$\gamma$-НСН & 0.94 & 1.00 & 0.96 & 0.58 & 0.95 & 1.00 & 0.97 & 1.00 & 0.99 & 0.98 & 0.98 & 0.97 & 0.95 \\
\hline p,p'-DDE & 0.003 & 0.05 & 0.07 & 0.003 & 0.01 & 0.14 & 0.11 & 0.17 & 0.01 & 0.21 & 0.07 & 0.16 & 0.30 \\
\hline o,p'-DDT & 0.004 & 0.001 & 0.004 & 0.000 & 0.08 & 0.000 & - & 0.002 & - & - & - & - & - \\
\hline p,p'-DDT & 0.15 & 0.07 & 0.31 & 0.14 & 0.55 & 0.41 & 0.17 & 0.49 & - & - & 0.04 & 0.05 & - \\
\hline \multicolumn{14}{|c|}{ 2008-2009 } \\
\hline & GBJD & Lulang & Bomi & Rawu & Qamdo & Lhasa & Namco & Lhaze & Xigaze & Everest & Saga & Naqu & Gar \\
\hline HCB & - & 0.78 & 0.79 & 0.81 & 0.92 & 0.88 & 0.30 & 0.94 & 0.80 & 0.81 & 0.88 & 0.86 & 0.85 \\
\hline PCB-28 & - & 0.92 & 0.94 & 0.69 & 0.97 & 1.00 & 0.86 & 1.00 & 1.00 & 0.99 & 0.99 & 0.99 & 1.00 \\
\hline PCB-52 & - & 0.82 & 0.95 & 0.22 & 0.80 & 0.99 & 0.09 & 0.79 & - & - & 0.55 & 0.38 & - \\
\hline$\alpha-H C H$ & - & 0.82 & 0.70 & 0.82 & 0.95 & 0.88 & 0.70 & 0.98 & 0.53 & 0.86 & 0.69 & 0.59 & 0.86 \\
\hline$\gamma-\mathbf{H C H}$ & - & 0.99 & 0.96 & 0.99 & 0.97 & 0.99 & 0.96 & 1.00 & 0.98 & 0.99 & 0.99 & 0.97 & 0.99 \\
\hline p,p'-DDE & - & 0.26 & 0.08 & 0.06 & 0.14 & 0.56 & 0.08 & 0.35 & 0.05 & 0.14 & 0.39 & 0.58 & 0.51 \\
\hline o,p'-DDT & - & 0.01 & 0.01 & 0.01 & 0.91 & 0.003 & - & 0.01 & - & - & - & - & - \\
\hline p,p'-DDT & - & 0.11 & 0.07 & 0.43 & 0.87 & 0.70 & 0.12 & 0.56 & - & - & 0.14 & 0.15 & - \\
\hline \multicolumn{14}{|c|}{ 2009-2010 } \\
\hline HCB & GBJD & Lulang & Bomi & Rawu & Qamdo & Lhasa & Namco & Lhaze & Xigaze & Everest & Saga & Naqu & Gar \\
\hline PCB-28 & - & 0.77 & 0.93 & 0.70 & 0.92 & 0.95 & 0.42 & 0.96 & 0.83 & 0.88 & 0.90 & 0.83 & 0.92 \\
\hline PCB-52 & - & 0.91 & 0.90 & 0.72 & 0.97 & 1.00 & 0.95 & 1.00 & 1.00 & 1.00 & 1.00 & 0.99 & 1.00 \\
\hline$\alpha-\mathrm{HCH}$ & - & 0.96 & 1.00 & 0.45 & 0.93 & 1.00 & 0.35 & 0.98 & - & - & 0.91 & 0.79 & - \\
\hline
\end{tabular}




\begin{tabular}{|c|c|c|c|c|c|c|c|c|c|c|c|c|c|}
\hline$\gamma-\mathbf{H C H}$ & - & 0.80 & 0.82 & 0.72 & 0.93 & 0.95 & 0.73 & 0.98 & 0.78 & 0.88 & 0.77 & 0.58 & 0.97 \\
\hline p,p'-DDE & - & 0.99 & 0.98 & 0.97 & 0.95 & 1.00 & 0.97 & 1.00 & 1.00 & 1.00 & 0.99 & 0.97 & 1.00 \\
\hline o,p'-DDT & - & 0.11 & 0.02 & 0.01 & 0.09 & 0.28 & 0.02 & 0.15 & 0.02 & 0.04 & 0.21 & 0.17 & 0.46 \\
\hline \multirow[t]{2}{*}{ p,p'-DDT } & - & 0.002 & 0.002 & 0.001 & 0.54 & 0.001 & - & 0.01 & - & - & - & - & - \\
\hline & - & 0.10 & 0.07 & 0.30 & 0.84 & 0.58 & 0.02 & 0.49 & - & - & 0.09 & 0.15 & - \\
\hline \multicolumn{14}{|c|}{ 2010-2011 } \\
\hline & GBJD & Lulang & Bomi & Rawu & Qamdo & Lhasa & Namco & Lhaze & Xigaze & Everest & Saga & Naqu & Gar \\
\hline HCB & 0.83 & 0.78 & 0.95 & 0.80 & 0.92 & 0.97 & 0.54 & 0.96 & 0.79 & 0.82 & 0.84 & 0.84 & 0.94 \\
\hline РCB-28 & 0.82 & 0.84 & 0.94 & 0.28 & 0.98 & 1.00 & 0.77 & 1.00 & 0.99 & 0.99 & 0.99 & 0.99 & 1.00 \\
\hline PCB-52 & 0.99 & 0.97 & 1.00 & 0.49 & 0.98 & 1.00 & 0.59 & 0.98 & - & - & 0.93 & 0.84 & - \\
\hline$\alpha-H C H$ & 0.44 & 0.89 & 0.88 & 0.84 & 0.96 & 0.98 & 0.87 & 0.99 & 0.80 & 0.93 & 0.71 & 0.69 & 0.98 \\
\hline$\gamma-\mathbf{H C H}$ & 0.98 & 1.00 & 0.99 & 0.99 & 0.99 & 1.00 & 0.99 & 1.00 & 1.00 & 1.00 & 0.99 & 0.99 & 1.00 \\
\hline p,p'-DDE & 0.02 & 0.25 & 0.07 & 0.02 & 0.29 & 0.50 & 0.12 & 0.54 & 0.04 & 0.11 & 0.43 & 0.50 & 0.81 \\
\hline o,p'-DDT & 0.04 & 0.01 & 0.02 & 0.01 & 0.88 & 0.01 & - & 0.07 & - & - & - & - & - \\
\hline p,p'-DDT & 0.44 & 0.38 & 0.55 & 0.76 & 0.99 & 0.89 & 0.17 & 0.94 & - & - & 0.30 & 0.63 & - \\
\hline \multicolumn{14}{|c|}{ 2011-2012 } \\
\hline & GBJD & Lulang & Bomi & Rawu & Qamdo & Lhasa & Namco & Lhaze & Xigaze & Everest & Saga & Naqu & Gar \\
\hline HCB & 0.87 & 0.84 & 0.97 & 0.87 & 0.97 & 0.98 & 0.51 & 0.98 & 0.87 & 0.89 & 0.93 & 0.83 & 0.96 \\
\hline PCB-28 & 0.91 & 0.96 & 0.99 & 0.84 & 0.99 & 1.00 & 0.98 & 1.00 & 1.00 & 1.00 & 1.00 & 0.99 & 1.00 \\
\hline PCB-52 & 0.99 & 0.97 & 1.00 & 0.57 & 0.99 & 1.00 & 0.67 & 0.99 & - & - & 0.95 & 0.78 & - \\
\hline$\alpha-H C H$ & 0.57 & 0.87 & 0.96 & 0.88 & 0.98 & 0.98 & 0.86 & 0.99 & 0.86 & 0.94 & 0.88 & 0.69 & 0.98 \\
\hline$\gamma$-НСН & 0.99 & 1.00 & 1.00 & 0.99 & 0.99 & 1.00 & 0.99 & 1.00 & 1.00 & 1.00 & 1.00 & 0.98 & 1.00 \\
\hline p,p'-DDE & 0.02 & 0.22 & 0.10 & 0.02 & 0.21 & 0.43 & 0.11 & 0.41 & 0.08 & 0.10 & 0.64 & 0.35 & 0.83 \\
\hline o,p'-DDT & 0.02 & 0.004 & 0.01 & 0.002 & 0.78 & 0.004 & - & 0.02 & - & - & - & - & - \\
\hline p,p'-DDT & 0.05 & 0.16 & 0.28 & 0.45 & 0.94 & 0.75 & 0.15 & 0.74 & - & - & 0.48 & 0.17 & - \\
\hline
\end{tabular}


Table S9. The soil-air net volatilization fluxes (ng/ $\mathrm{m}^{2} / \mathrm{h}$ ) of PCB-28, PCB-52, HCB, $\alpha-\mathrm{HCH}$ and $\gamma-\mathrm{HCH}$ at the Tibetan Plateau sites. (air data from 2007-2008 PAS)

\begin{tabular}{|c|c|c|c|c|c|c|c|c|c|c|c|c|c|}
\hline \multicolumn{14}{|c|}{ 2007-2008 } \\
\hline & GBJD & Lulang & Bomi & Rawu & Qamdo & Lhasa & Nam co & Lhaze & Xigaze & Everest & Saga & Naqu & Gar \\
\hline НСВ & -0.098 & -0.035 & -0.142 & -0.063 & -0.419 & -0.495 & -0.026 & -0.840 & -0.055 & -0.271 & -0.216 & -0.118 & -0.538 \\
\hline PCB-28 & -0.001 & 0.000 & -0.005 & 0.009 & -0.018 & -0.352 & -0.004 & -0.390 & -0.089 & -0.039 & -0.077 & -0.032 & -0.176 \\
\hline PCB-52 & -0.015 & -0.004 & -0.042 & 0.001 & -0.008 & -0.123 & 0.000 & -0.008 & - & - & -0.001 & 0.000 & - \\
\hline$\alpha-\mathbf{H C H}$ & 0.018 & -0.044 & -0.110 & 0.036 & -0.163 & -0.111 & -0.027 & -0.612 & -0.012 & -0.089 & -0.009 & -0.014 & -0.120 \\
\hline$\gamma-\mathbf{H C H}$ & -0.147 & -0.501 & -0.400 & -0.031 & -0.092 & -1.557 & -0.097 & -2.799 & -0.457 & -1.103 & -0.330 & -0.263 & -1.042 \\
\hline \multicolumn{14}{|c|}{$2008-2009$} \\
\hline & GBJD & Lulang & Bomi & Rawu & Qamdo & Lhasa & Nam co & Lhaze & $\overline{\text { Xigaze }}$ & Everest & Saga & Naqu & Gar \\
\hline HCB & - & -0.086 & -0.342 & -0.085 & -0.455 & -0.655 & 0.049 & -0.875 & -0.129 & -0.271 & -0.255 & -0.214 & -0.474 \\
\hline PCB-28 & - & -0.003 & -0.009 & 0.000 & -0.022 & -0.353 & -0.003 & -0.392 & -0.091 & -0.047 & -0.078 & -0.033 & -0.180 \\
\hline PCB-52 & - & -0.005 & -0.042 & 0.000 & -0.007 & -0.122 & 0.002 & -0.006 & - & - & 0.000 & 0.000 & - \\
\hline$\alpha-H C H$ & - & -0.041 & -0.081 & -0.023 & -0.160 & -0.112 & -0.016 & -0.604 & -0.003 & -0.088 & -0.015 & -0.015 & -0.114 \\
\hline$\gamma$-HCH & - & -0.500 & -0.401 & -0.105 & -0.094 & -1.553 & -0.096 & -2.799 & -0.453 & -1.118 & -0.333 & -0.264 & -1.090 \\
\hline \multicolumn{14}{|c|}{$2009-2010$} \\
\hline & GBJD & Lulang & Bomi & Rawu & Qamdo & Lhasa & Nam co & Lhaze & Xigaze & Everest & Saga & Naqu & Gar \\
\hline HCB & - & -0.084 & -0.428 & -0.063 & -0.452 & -0.718 & 0.014 & -0.898 & -0.135 & -0.309 & -0.263 & -0.202 & -0.522 \\
\hline PCB-28 & - & -0.003 & -0.009 & 0.000 & -0.023 & -0.354 & -0.004 & -0.392 & -0.091 & -0.047 & -0.078 & -0.033 & -0.181 \\
\hline PCB-52 & - & -0.006 & -0.044 & 0.000 & -0.008 & -0.123 & 0.000 & -0.009 & - & - & -0.002 & -0.001 & - \\
\hline$\alpha-H C H$ & - & -0.039 & -0.112 & -0.018 & -0.157 & -0.122 & -0.017 & -0.605 & -0.019 & -0.092 & -0.020 & -0.013 & -0.131 \\
\hline$\gamma-\mathbf{H C H}$ & - & -0.500 & -0.409 & -0.103 & -0.093 & -1.561 & -0.097 & -2.798 & -0.460 & -1.120 & -0.334 & -0.264 & -1.093 \\
\hline \multicolumn{14}{|c|}{$2010-2011$} \\
\hline
\end{tabular}




\begin{tabular}{|c|c|c|c|c|c|c|c|c|c|c|c|c|c|}
\hline & GBJD & Lulang & Bomi & Rawu & Qamdo & Lhasa & Nam co & Lhaze & Xigaze & Everest & Saga & Naqu & Gar \\
\hline НCB & -0.162 & -0.085 & -0.435 & -0.084 & -0.457 & -0.735 & -0.006 & -0.900 & -0.124 & -0.277 & -0.239 & -0.206 & -0.538 \\
\hline PCB-28 & -0.002 & -0.002 & -0.009 & 0.001 & -0.023 & -0.354 & -0.003 & -0.392 & -0.091 & -0.047 & -0.078 & -0.033 & -0.180 \\
\hline PCB-52 & -0.015 & -0.006 & -0.044 & 0.000 & -0.008 & -0.123 & 0.000 & -0.009 & - & - & -0.002 & -0.001 & - \\
\hline$\alpha-\mathbf{H C H}$ & 0.003 & -0.046 & -0.124 & -0.024 & -0.162 & -0.126 & -0.023 & -0.614 & -0.020 & -0.098 & -0.016 & -0.028 & -0.133 \\
\hline$\gamma-\mathbf{H C H}$ & -0.153 & -0.503 & -0.416 & -0.105 & -0.097 & -1.562 & -0.099 & -2.802 & -0.461 & -1.122 & -0.334 & -0.269 & -1.096 \\
\hline \multicolumn{14}{|c|}{ 2011-2012 } \\
\hline & GBJD & Lulang & Bomi & Rawu & Qamdo & Lhasa & Nam co & Lhaze & Xigaze & Everest & Saga & Naqu & Gar \\
\hline HCB & -0.171 & -0.097 & -0.448 & -0.094 & -0.479 & -0.743 & -0.002 & -0.917 & -0.145 & -0.314 & -0.274 & -0.202 & -0.548 \\
\hline PCB-28 & -0.002 & -0.003 & -0.010 & 0.000 & -0.023 & -0.354 & -0.004 & -0.392 & -0.092 & -0.048 & -0.078 & -0.033 & -0.181 \\
\hline PCB-52 & -0.015 & -0.006 & -0.044 & 0.000 & -0.009 & -0.123 & 0.000 & -0.009 & - & - & -0.002 & -0.001 & - \\
\hline$\alpha-\mathrm{HCH}$ & -0.003 & -0.045 & -0.138 & -0.026 & -0.165 & -0.127 & -0.023 & -0.615 & -0.022 & -0.100 & -0.024 & -0.028 & -0.133 \\
\hline$\gamma-\mathbf{H C H}$ & -0.155 & -0.502 & -0.417 & -0.105 & -0.097 & -1.562 & -0.099 & -2.802 & -0.461 & -1.122 & -0.335 & -0.267 & -1.095 \\
\hline
\end{tabular}


Table SI-10. Relative composition data (\%) used for cluster analysis

\begin{tabular}{|c|c|c|c|c|c|c|c|c|}
\hline Samples & $\alpha-H C H$ & $\gamma-\mathbf{H C H}$ & HCB & o,p'-DDE & p,p'-DDE & o,p'-DDT & p,p'-DDT & $\sum_{6} \mathrm{PCBs}$ \\
\hline Xigaze 1 & 4.1 & 1.6 & 32.3 & 4.1 & 6.4 & 34.0 & 14.0 & 3.7 \\
\hline Namco 1 & 1.0 & 6.3 & 26.2 & 24.1 & 1.9 & 30.4 & 5.2 & 4.7 \\
\hline Lhasa 1 & 4.1 & 1.4 & 56.4 & 2.2 & 5.7 & 22.4 & 6.2 & 1.5 \\
\hline Lhaze 1 & 4.1 & 2.0 & 56.1 & 2.5 & 2.5 & 23.9 & 6.4 & 2.4 \\
\hline Lulang 1 & 2.6 & 0.8 & 27.0 & 4.6 & 6.2 & 45.6 & 10.4 & 2.8 \\
\hline Everest 1 & 11.8 & 14.1 & 57.6 & 0.3 & 0.8 & 8.1 & 1.3 & 6.0 \\
\hline Naqu 1 & 12.2 & 3.2 & 45.8 & 2.8 & 1.3 & 25.6 & 6.8 & 2.3 \\
\hline Qamdo 1 & 1.5 & 1.3 & 19.5 & 8.1 & 13.3 & 41.7 & 11.4 & 3.1 \\
\hline Saga 1 & 6.6 & 2.2 & 27.2 & 4.3 & 7.7 & 38.4 & 12.1 & 1.6 \\
\hline Rawu 1 & 16.3 & 18.5 & 11.7 & 1.1 & 4.1 & 38.6 & 7.1 & 2.6 \\
\hline Bomi 1 & 6.9 & 3.8 & 64.4 & 2.1 & 4.5 & 12.2 & 3.2 & 3.0 \\
\hline GBJD 1 & 8.8 & 2.7 & 29.3 & 4.3 & 8.6 & 35.7 & 8.3 & 2.4 \\
\hline Golmud 1 & 5.4 & 1.9 & 82.3 & 2.8 & 2.2 & 2.6 & 0.9 & 1.8 \\
\hline Gar 1 & 9.1 & 31.9 & 20.6 & 2.2 & 4.7 & 22.8 & 5.2 & 3.5 \\
\hline Chayu 2 & 7.7 & 1.9 & 36.9 & 0.3 & 2.5 & 13.7 & 35.7 & 1.3 \\
\hline Bomi 2 & 17.1 & 4.8 & 32.9 & 0.7 & 5.0 & 11.8 & 26.7 & 1.1 \\
\hline Xigaze 2 & 22.0 & 8.6 & 40.1 & 3.2 & 4.4 & 8.5 & 10.9 & 2.3 \\
\hline Namco 2 & 10.2 & 3.7 & 74.6 & 0.9 & 1.0 & 3.8 & 2.8 & 3.1 \\
\hline Lhasa 2 & 10.5 & 6.2 & 63.5 & 0.3 & 2.0 & 10.3 & 5.2 & 2.0 \\
\hline Lhaze 2 & 14.2 & 3.5 & 60.7 & 0.5 & 1.7 & 7.4 & 8.1 & 4.0 \\
\hline Lulang 2 & 11.7 & 3.5 & 34.2 & 3.4 & 3.2 & 18.8 & 22.6 & 2.5 \\
\hline Everest 2 & 11.6 & 4.0 & 56.3 & 0.3 & 1.3 & 13.6 & 11.0 & 1.8 \\
\hline Naqu 2 & 36.1 & 8.7 & 41.2 & 0.5 & 0.6 & 3.9 & 7.0 & 2.2 \\
\hline Qamdo 2 & 11.6 & 4.6 & 57.7 & 0.8 & 6.6 & 2.1 & 11.2 & 5.4 \\
\hline Saga 2 & 16.0 & 4.5 & 50.0 & 0.5 & 3.3 & 10.8 & 11.7 & 3.1 \\
\hline Rawu 2 & 14.6 & 3.0 & 56.0 & 1.3 & 1.5 & 6.2 & 14.0 & 3.4 \\
\hline Golmud 2 & 11.3 & 5.4 & 79.4 & 0.4 & 0.4 & 0.2 & 0.2 & 2.7 \\
\hline Gar 2 & 15.3 & 4.6 & 70.6 & 1.5 & 2.3 & 0.2 & 2.5 & 2.9 \\
\hline Xigaze 3 & 6.1 & 1.9 & 29.4 & 9.4 & 14.0 & 26.8 & 11.5 & 0.9 \\
\hline Namco 3 & 7.6 & 2.0 & 38.7 & 1.6 & 3.5 & 27.8 & 15.4 & 3.3 \\
\hline Lhasa 3 & 6.4 & 2.0 & 37.0 & 1.6 & 9.8 & 28.6 & 12.8 & 1.9 \\
\hline Lhaze 3 & 14.6 & 5.0 & 43.4 & 0.4 & 5.9 & 17.1 & 12.1 & 1.5 \\
\hline Lulang 3 & 9.5 & 2.3 & 26.0 & 1.1 & 6.3 & 35.8 & 17.8 & 1.3 \\
\hline Everest 3 & 10.7 & 3.2 & 37.0 & 1.5 & 5.1 & 28.8 & 12.5 & 1.2 \\
\hline Naqu 3 & 29.7 & 6.7 & 42.2 & 0.3 & 2.9 & 11.9 & 5.4 & 0.9 \\
\hline Qamdo 3 & 12.3 & 4.8 & 47.6 & 0.9 & 7.6 & 13.3 & 11.0 & 2.4 \\
\hline Saga 3 & 10.3 & 2.7 & 39.1 & 0.4 & 7.7 & 19.2 & 19.3 & 1.3 \\
\hline
\end{tabular}




\begin{tabular}{|c|c|c|c|c|c|c|c|c|}
\hline Rawu 3 & 11.7 & 2.9 & 47.2 & 0.7 & 4.0 & 21.6 & 11.0 & 0.9 \\
\hline Bomi 3 & 8.5 & 2.6 & 9.0 & 1.5 & 18.5 & 32.5 & 26.6 & 0.8 \\
\hline Golmud 3 & 12.0 & 3.2 & 66.4 & 0.4 & 2.4 & 10.3 & 3.5 & 1.9 \\
\hline Gar 3 & 6.2 & 4.2 & 64.5 & 0.5 & 4.9 & 7.3 & 9.6 & 2.8 \\
\hline Chayu 3 & 7.1 & 2.0 & 28.5 & 1.5 & 7.9 & 33.9 & 18.3 & 0.8 \\
\hline Muztaga 3 & 8.2 & 1.7 & 73.3 & 0.5 & 4.5 & 4.5 & 5.9 & 1.4 \\
\hline Xigaze 4 & 9.6 & 1.4 & 69.0 & 3.2 & 8.4 & 5.5 & 1.1 & 1.8 \\
\hline Namco 4 & 9.3 & 1.4 & 70.3 & 0.8 & 1.6 & 6.8 & 5.2 & 4.4 \\
\hline Lhasa 4 & 7.8 & 1.2 & 56.1 & 2.6 & 10.5 & 12.3 & 6.3 & 3.3 \\
\hline Lhaze 4 & 9.9 & 1.4 & 79.5 & 0.8 & 1.6 & 2.9 & 1.4 & 2.5 \\
\hline Lulang 4 & 10.7 & 1.2 & 55.9 & 1.8 & 5.4 & 15.6 & 7.0 & 2.3 \\
\hline Everest 4 & 7.7 & 1.9 & 74.8 & 0.9 & 2.5 & 8.0 & 2.9 & 1.2 \\
\hline Naqu 4 & 28.2 & 5.0 & 59.8 & 0.5 & 0.9 & 3.3 & 0.8 & 1.4 \\
\hline Qamdo 4 & 11.3 & 1.8 & 74.9 & 0.8 & 3.4 & 3.7 & 1.5 & 2.6 \\
\hline Saga 4 & 14.7 & 2.3 & 69.8 & 0.4 & 2.9 & 4.1 & 4.5 & 1.3 \\
\hline Rawu 4 & 12.8 & 2.8 & 59.4 & 1.8 & 4.6 & 10.5 & 3.4 & 4.8 \\
\hline Bomi 4 & 22.2 & 3.4 & 29.3 & 1.5 & 24.0 & 11.4 & 6.5 & 1.8 \\
\hline GBJD 4 & 18.0 & 4.2 & 45.4 & 1.3 & 5.4 & 16.5 & 7.5 & 1.6 \\
\hline Golmud 4 & 5.7 & 0.8 & 88.4 & 0.4 & 0.9 & 1.5 & 0.8 & 1.5 \\
\hline Gar 4 & 6.3 & 1.0 & 83.9 & 0.9 & 1.9 & 1.4 & 1.7 & 2.9 \\
\hline Chayu 4 & 4.4 & 0.5 & 30.2 & 2.2 & 16.6 & 27.0 & 18.0 & 1.0 \\
\hline Muztaga 4 & 7.3 & 0.7 & 86.2 & 0.3 & 3.1 & 0.5 & 0.6 & 1.2 \\
\hline Xigaze 5 & 9.5 & 2.3 & 57.6 & 2.7 & 6.7 & 13.4 & 6.6 & 1.2 \\
\hline Namco 5 & 8.8 & 2.3 & 67.5 & 0.6 & 1.5 & 13.3 & 5.0 & 1.0 \\
\hline Lhasa 5 & 5.7 & 1.3 & 34.5 & 2.9 & 13.2 & 24.6 & 15.6 & 2.2 \\
\hline Lhaze 5 & 11.2 & 2.0 & 56.1 & 1.0 & 3.6 & 14.7 & 10.0 & 1.5 \\
\hline Lulang 5 & 10.2 & 2.4 & 30.3 & 1.7 & 5.2 & 31.0 & 18.3 & 0.9 \\
\hline Everest 5 & 8.2 & 2.6 & 54.5 & 1.0 & 3.4 & 18.7 & 10.4 & 1.2 \\
\hline Naqu 5 & 24.3 & 5.4 & 55.1 & 0.5 & 1.5 & 6.5 & 5.9 & 0.8 \\
\hline Qamdo 5 & 10.5 & 3.2 & 51.6 & 1.5 & 8.1 & 12.2 & 10.0 & 2.9 \\
\hline Saga 5 & 12.2 & 3.7 & 64.1 & 0.9 & 2.9 & 9.6 & 5.3 & 1.4 \\
\hline Rawu 5 & 9.8 & 2.6 & 40.1 & 1.7 & 4.7 & 25.3 & 14.4 & 1.4 \\
\hline Bomi 5 & 6.6 & 1.6 & 16.1 & 2.3 & 17.2 & 31.9 & 22.1 & 2.3 \\
\hline GBJD 5 & 5.5 & 1.2 & 18.6 & 0.7 & 2.9 & 12.9 & 57.4 & 0.7 \\
\hline Golmud 5 & 8.3 & 3.1 & 80.1 & 0.2 & 2.1 & 1.7 & 2.4 & 2.1 \\
\hline Gar 5 & 9.1 & 2.3 & 75.7 & 0.4 & 2.1 & 4.9 & 3.9 & 1.6 \\
\hline Chayu 5 & 6.2 & 1.2 & 24.3 & 1.6 & 9.3 & 31.1 & 25.3 & 0.9 \\
\hline Muztaga 5 & 8.0 & 1.2 & 78.2 & 0.1 & 4.3 & 2.8 & 4.6 & 0.9 \\
\hline
\end{tabular}

The naming convention of the samples is the site name plus the sampling year. For example, “Xigaze 1” is the sample for site of Xigaze in the first sampling year of 2007 to 2008. 
Table S11. Results of the grouping using cluster analysis

\begin{tabular}{|c|c|c|c|}
\hline 3 groups & Group 1 & Group 2 & Group 3 \\
\hline Number of sites & 28 & 17 & 30 \\
\hline \multirow{30}{*}{ Sample name } & Xigaze 1 & Golmud 1 & Lhasa 1 \\
\hline & Namco 1 & Namco 2 & Lhaze 1 \\
\hline & Lulang 1 & Golmud 2 & Everest 1 \\
\hline & Naqu 1 & Gar 2 & Bomi 1 \\
\hline & Qamdo 1 & Muztaga 3 & Xigaze 2 \\
\hline & Saga 1 & Xigaze 4 & Lhasa 2 \\
\hline & Rawu 1 & Namco 4 & Lhaze 2 \\
\hline & GBJD 1 & Lhaze 4 & Everest 2 \\
\hline & Gar 1 & Everest 4 & Naqu 2 \\
\hline & Lulang 2 & Qamdo 4 & Qamdo 2 \\
\hline & Chayu 2 & Saga 4 & Saga 2 \\
\hline & Bomi 2 & Muztaga 4 & Rawu 2 \\
\hline & Xigaze 3 & Golmud 4 & Lhaze 3 \\
\hline & Namco 3 & Gar 4 & Naqu 3 \\
\hline & Lhasa 3 & Golmud 5 & Qamdo 3 \\
\hline & Lulang 3 & Gar 5 & Golmud 3 \\
\hline & Everest 3 & Muztaga 5 & Gar 3 \\
\hline & Saga 3 & & Lhasa 4 \\
\hline & Chayu 3 & & Lulang 4 \\
\hline & Rawu 3 & & Naqu 4 \\
\hline & Bomi 3 & & Rawu 4 \\
\hline & Chayu 4 & & Bomi 4 \\
\hline & Lhasa 5 & & GBJD 4 \\
\hline & Lulang 5 & & Xigaze 5 \\
\hline & Chayu 5 & & Namco 5 \\
\hline & Rawu 5 & & Lhaze 5 \\
\hline & Bomi 5 & & Everest 5 \\
\hline & GBJD 5 & & Naqu 5 \\
\hline & & & Qamdo 5 \\
\hline & & & Saga 5 \\
\hline composition (\%) & Group 1 & Group 2 & Group 3 \\
\hline$\alpha-\mathrm{HCH}$ & $8.0(1.0-17.1)$ & $9.3(5.4-15.3)$ & $14.1(4.1-36.1)$ \\
\hline$\gamma-\mathbf{H C H}$ & $4.0(0.5-31.9)$ & $2.2(0.7-5.4)$ & $4.3(1.2-14.1)$ \\
\hline HCB & $29.6(9.0-47.2)$ & $77.1(69.0-88.4)$ & $54.6(29.3-67.5)$ \\
\hline DDTs & 56.5 (34.9-79.1) & $9.3(1.2-18.1)$ & $24.7(5.6-43.3)$ \\
\hline$\sum_{6}$ PCBs & $1.9(0.7-4.7)$ & $2.1(0.9-4.4)$ & $2.4(0.8-6.0)$ \\
\hline
\end{tabular}

The average and range (in brackets) of composition in each group were listed. 
Table S12. The representative sites in each group

\begin{tabular}{ccc}
\hline Group 1 & Group 2 & Group 3 \\
\hline Chayu (100\%) $^{\mathrm{a}}$ & Muztaga (100\%) & Lhaze (80\%) \\
Lulang (80\%) & Golmud (80\%) & Naqu (80\%) \\
GBJD (67\%) & Gar (60\%) & Everest (60\%) \\
Rawu (60\%) & & Qamdo (60\%) \\
Bomi (60\%) & & Lhasa (60\%) \\
\hline
\end{tabular}

a. The percentages in the brackets after the site name represent the frequency of the sampling site appears in this group, which is derived through dividing the times of the sampling site appears in this group to the total sampling times in this site.

b. Most sites have 5 samples of the consecutive 5 years, except that Chayu sampled for 4 years; GBJD sampled for 3 years and Muztaga for 3 years. (see Table S1)

c. Due to the possible local contamination of Naqu, Naqu was not included as a representative site of this group. 


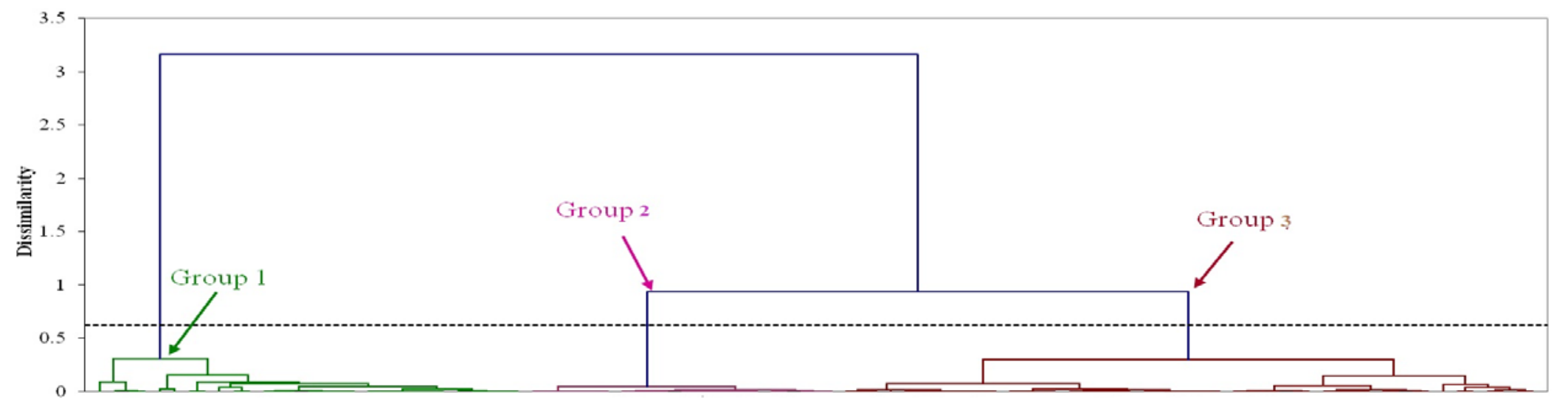

$=\alpha-\mathrm{HCH} \equiv \gamma-\mathrm{HCH} \equiv \mathrm{HCB}=\mathrm{o}, \mathrm{p}^{\prime}-\mathrm{DDE} \equiv \mathrm{p}, \mathrm{p}^{\prime}-\mathrm{DDE} \equiv \mathrm{o}, \mathrm{p}^{\prime}-\mathrm{DDT} \equiv \mathrm{p}, \mathrm{p}^{\prime}-\mathrm{DDT}=\sum 6 \mathrm{PCBs}$

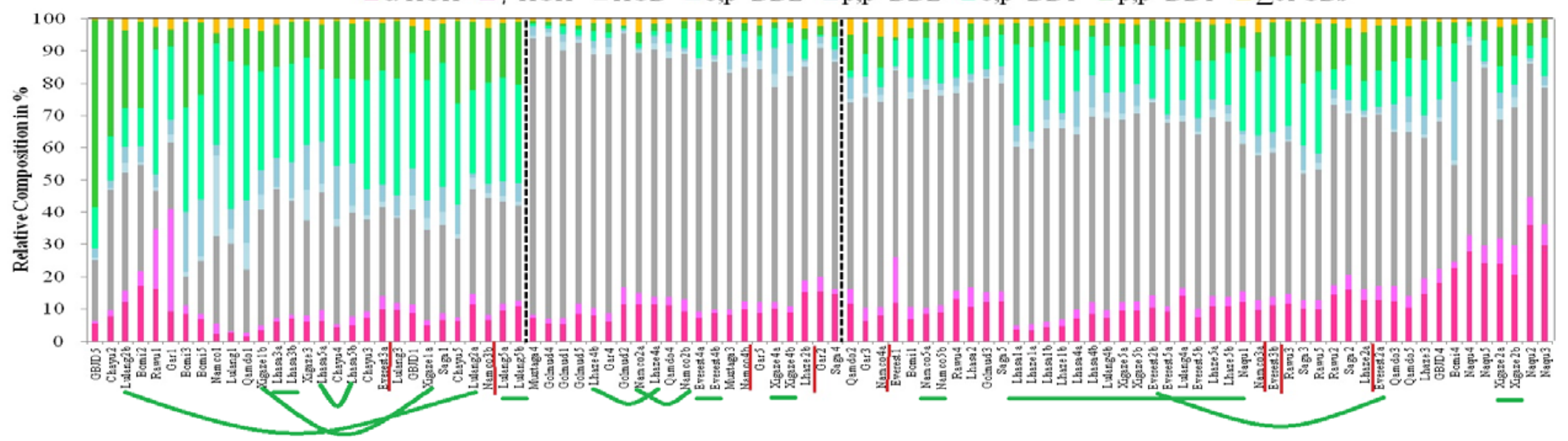

Figure S1 Result of cluster analysis after including the duplicate samples

In this study, 23 pairs of duplicates were obtained (Table S4). Duplicates were named by the name of sampling site followed by sampling year 
and alphabet a or b. For example, Lhasa 1a and Lhasa 1b means the Lhasa duplicates employed for the first sampling year (2007-2008) and Lhasa 2a and 2b means the Lhasa duplicates employed for the second sampling year (2008-2009) and so on. From the figure above, we found that most of samples appeared just shoulder by shoulder, such as the duplicates labeled by green horizontal lines, and the duplicates grouped in the same group were connected by green curves. The samples labeled with red vertical lines were duplicate samples (4 pairs) dispersed in different groups. From Liu and Wania (2014), they suggested only when less than $20 \%$ of the duplicates in a study fail to be in the same group, may the analytical uncertainties be acceptable for clustering results. In our study, only 4 of 23 pairs of duplicate samples were dispersed in different groups, which is less than the threshold of $20 \%$. This suggested that the results of cluster analysis were from the real difference/similarity between sampling sites but not the random error of the analytical procedure. 

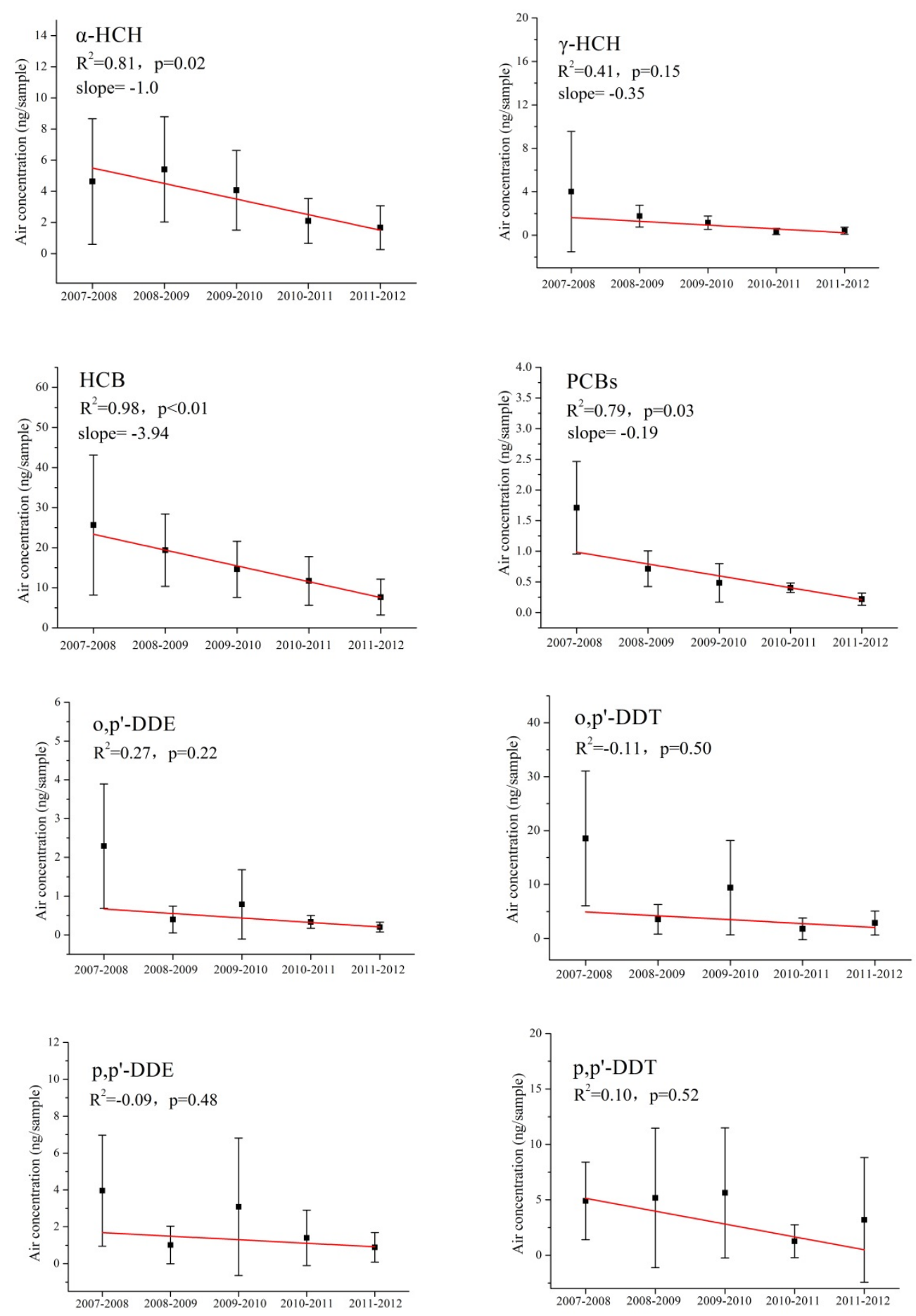

Figure S2 Inter-annual concentration variation from 2007 to 2012 
Table S13 . Results of randomized block ANOVA test for the concentrations of DDTs in the monsoon region, westerly region and transition region from 2007 to 2012. (p $<0.05$ indicates that the levels of DDTs significantly differ between two years.)

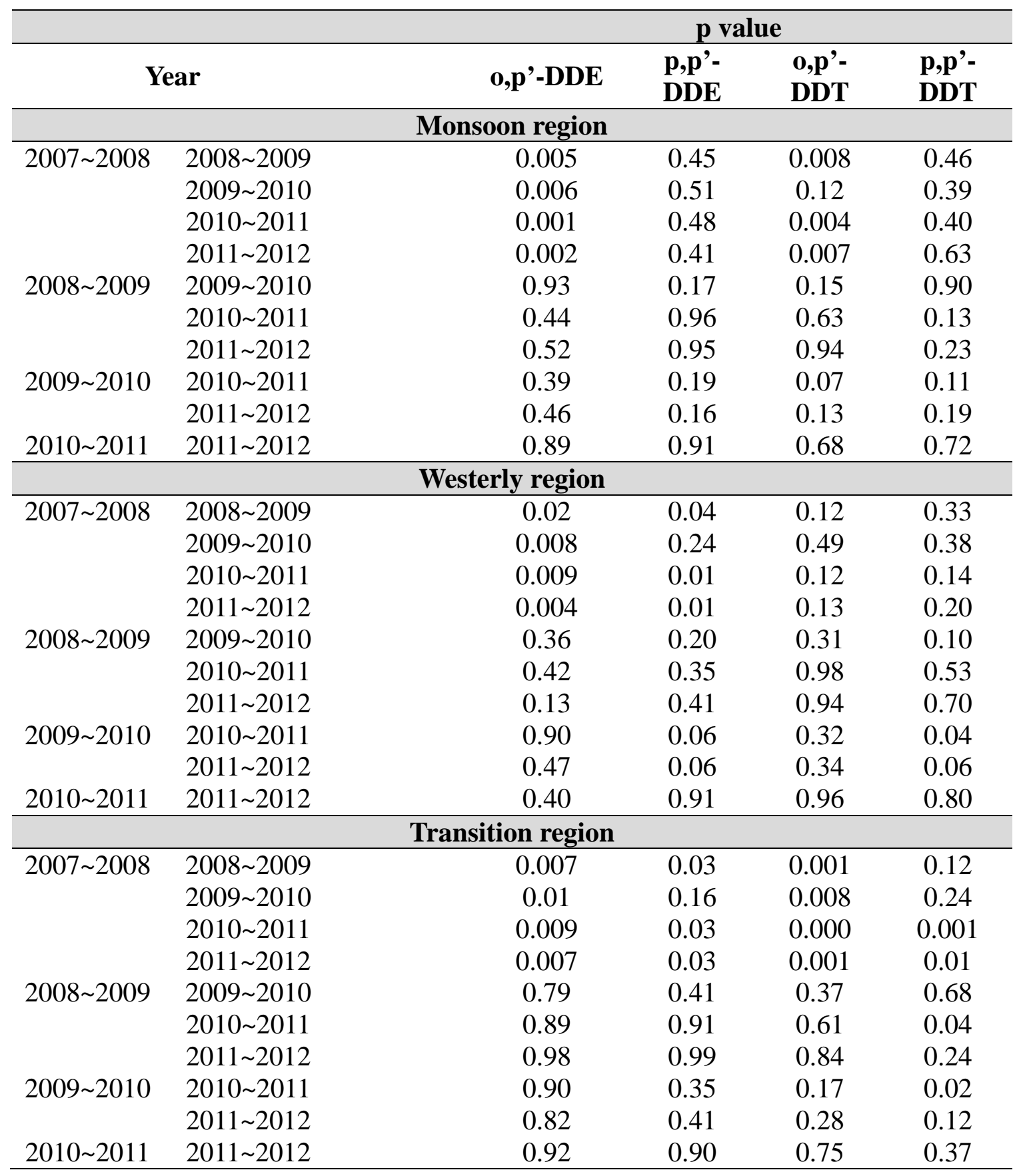




\section{References:}

Backe, C., Cousins, I.T., Larsson, P. PCB in soils and estimated soil-air exchange fluxes of selected PCB congeners in the South of Sweden. Environmental Pollution 2004, 128. 59-72.

Gouin, T., Harner, T., Blanchard, P., Passive and active air samplers as complementary methods for investigating persistent organic pollutants in the Great Lakes basin. Environmental science and technology, 2005, 39(23): 9115-9122.

Harner, T., Bidleman, T.F., Jantunen, L.M.M., Mackay, D., 2001. Soileair exchange model of persistent pesticides in the United States cotton belt. Environmental Toxicology and Chemistry 20, 1612-1621.

Li, Y.F., Harner, T., Liu, L., Zhang, Z., Ren, N.-Q., Jia, H., Ma, J., Sverko, E., 2009. Polychlorinated biphenyls in global air and surface soil: distributions, air-soil exchange, and fractionation effect? Environmental Science and Technology, 44, 2784-2790.

Mackay, D. Multimedia Environmental Models: The Fugacity Approach; Lewis: Boca Raton FL, 1991.

McLachlan M.S., Czub, G., Wania, F., 2002. The influence of vertical sorbed phase transport on the fate of organic chemicals in surface soils. Environmental Science and Technology 36, $4860-4867$.

Sheng, J., Wang, X., Gong, P., Joswiak, D. R.; Tian, L.;Yao, T. D.; Jones, K. C. Monsoon-driven transport of organochlorine pesticides and polychlorinated biphenyls to the Tibetan Plateau: three year atmospheric monitoring study. Environmental science and technology, 2013, 47(7): 3199-3208.

Shunthirasingham, C., Oyiliagu, C. E., Cao, X., Gouin, T., Wania, F., Lee, S.-C., Pozo, K., Harner, T., and Muir, D. C.: Spatial and temporal pattern of pesticides in the global atmosphere, Journal of Environmental Monitoring, 12, 1650-1657, 2010.

Wang, X. P.; Gong, P.; Yao, T. D.; Jones, K. C. Passive air sampling of organochlorine pesticides, polychlorinated biphenyls, and polybrominated diphenyl ethers across the Tibetan Plateau. Environmental science and technology, 2010, 44 (8), 2988-2993. 